\title{
ABIN-1 in the brain alleviates opioid tolerance by interacting with $\beta$-arrestin2 and MOR
}

\author{
Ruibin $\mathrm{Su}^{1}$, Yixin Zhang ${ }^{1}$, Peilan Zhou ${ }^{2}$, Zehui Gong ${ }^{2}$, and Fengfeng $\mathrm{Lu}^{1}$ \\ ${ }^{1}$ Beijing Institute of Pharmacology and Toxicology \\ ${ }^{2}$ Beijing Institute of Pharmacology \& Toxicology
}

May 5, 2020

\begin{abstract}
Background: While opioids play a crucial role in pain's relief, chronic exposure results in tolerance and dependence. Efforts should be made to alleviate the side effect induced by opioids. Many proteins which functionally interact with MOR can regulate the effect of opioids. Our bacterial yeast two-hybrid experiment showed ABIN-1 could bind to MOR. Here, we studied the profile and mechanism of ABIN-1 on morphine tolerance and dependence. Experimental Approach: ABIN-1 in mouse brain was interfered by AAV virus. The tolerance and dependence induced by morphine were assessed in hotplate and conditioned place preference test. The regulation of $\beta$-arrestin signalling of MOR was observed in MOR-CHO cell lines after ABIN-1 overexpression. The interaction of proteins was detected by co-immunoprecipitation and immunofluorescence. The expression of proteins was tested by western blotting and immunohistochemistry. Key Results: Morphine tolerance and dependence were attenuated by overexpression of ABIN-1 in mouse brains. ABIN-1 in the hippocampus and nucleus accumbens participated in morphine tolerance and physical dependence. MOR phosphorylation and internalization were weakened by ABIN-1 after opioids treatment. Formation of ABIN-1- $\beta$-arrestin- 2 complexes promoted the translocation of $\beta$-arrestin- 2 to the plasma membrane and accelerated its ubiquitination and degradation. Furthermore, attenuation of morphine tolerance by ABIN-1 was abolished in $\beta$-arrestin-2 knockout mice. Conclusions and Implications: These findings indicate that ABIN-1 co-operates with $\beta$-arrestin2 and MOR to alleviate morphine tolerance and dependence. ABIN-1 may be a target to alleviate morphine tolerance.
\end{abstract}

\section{What is already known}

* ABIN-1 could interaction with MOR.

\section{What this study adds}

* ABIN-1 in mouse brains attenuated morphine tolerance and dependence

* Formation of ABIN-1- $\beta$-arrestin- 2 complexes promoted the translocation of $\beta$-arrestin- 2 to the plasma membrane and accelerated degradation

\section{What is the clinical significance}

* Our data provide a new perspective target on alleviating morphine tolerance and dependence

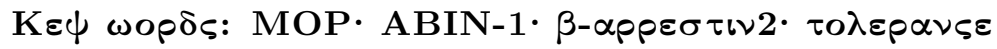

\section{Introduction}

Opioids, such as morphine, have powerful analgesic effects to relieve various pains, but their repeated or continuous use can result in tolerance and dependence. These side effects limit the clinical utility of morphine. The activation of $\mu$ opioid receptor (MOR) is potential mechanism of tolerance, include G protein-dependent 
signalling and $\beta$-arrestin-mediated signalling(Al-Hasani \& Bruchas, 2011). Super-activation or sensitization of adenylate cyclase (AC) and internalization of MOR by chronic agonist treatment are considered as the critical mechanisms of opioid tolerance, but further mechanisms have not been elucidated(Sharma S K 1975).

$\beta$-arrestin-mediated signalling of MOR has great effects on morphine tolerance(Molinari et al., 2010). Agonist-induced phosphorylation of MOR is thought to be a trigger for $\beta$-arrestin-mediated signalling. In MOR phosphorylation-deficient mice, opioid-induced analgesia was strongly enhanced and tolerance was greatly diminished(Kliewer et al., 2019). $\beta$-arrestins, including $\beta$-arrestin1 and $\beta$-arrestin2, are highly expressed in the central nervous system (CNS) and play critical roles in regulating MOR(Attramadal et al., 1992). In cells, $\beta$-arrestin2 is recruited after MOR phosphorylation, which cause sequestration of MOR under morphine exposure(DeWire, Ahn, Lefkowitz \& Shenoy, 2007). Besides, ubiquitinated $\beta$-arrestins are crucial for downstream endocytic and signalling processes(Takenouchi, Yoshimura \& Ozawa, 2018). Mice lacking $\beta$-arrestin2 did not develop antinociceptive tolerance in a hot plate test following chronic morphine treatment(Raehal \& Bohn, 2011). Several proteins have been found to mediate morphine tolerance by regulating $\beta$-arrestin signalling. For example, TRPV1 promoted rapid translocation of $\beta$-arrestin2 to the nucleus and decreased the desensitization of MOR to enhance analgesia(Basso Lilian, 20). The vasopressin $1 \mathrm{~b}$ receptor enhanced morphine analgesia by interaction between $\beta$-arrestin2 and MOR(Koshimizu et al., 2018). MiR-365 reduced morphine analgesic tolerance by influencing $\beta$-arrestin2 negatively $(\mathrm{Wu}$, She, Yang, Xing, Chen \& Zhang, 2018). Therefore, MOR-associated proteins that bias MOR towards the Gi signalling pathway are thought to be therapeutic targets and molecular probes to resolve the limitations of morphine(Inagaki et al., 2015). Recent progress has supported the feasibility and potential clinical utility of biased MOR agonists such as PZM21 and TRV130(Manglik et al., 2016) (Heyninck, Kreike \& Beyaert, 2003) .

Our previous work demonstrated that ABIN-1 interacted with MOR(Zhou, 2017). Although ABIN-1 is well known as a ubiquitin-binding protein that regulates processes in autoimmune inflammatory diseases such as systemic lupus erythematosus (SLE) and psoriasis, its role in the brain has rarely been reported (Nanda et al., 2011). Therefore, we aimed to explore the role of ABN-1 in the CNS on morphine tolerance and investigate the underlying mechanisms.

In the present study, we showed that ABIN-1 overexpression in mice brain attenuates morphine tolerance and dependence, and ABIN-1 in both the hippocampus (Hipp) and nucleus accumbens (NAc) decreased morphine-induced tolerance and physical dependence. ABIN-1 decreased MOR phosphorylation and internalization under opioid treatment and increased the translocation of $\beta$-arrestin2 to the plasma membrane and ubiquitinated it by interaction. As a result, ABIN-1 had no effect on morphine tolerance in $\beta$-arrestin2knockout mice. Our data uncovered an unappreciated but meaningful mechanism by which ABIN-1 decreases opioid tolerance by co-operating with $\beta$-arrestin2 and MOR. Therefore, ABIN-1 could be a new target to regulate morphine tolerance.

\section{Methods}

\subsection{Animals}

Female C57BL/6 mice aged 8 weeks $(18-22$ g) were used in all experiments. $\beta$-arrestin2-knockout (ARRB2 $2^{-/}$) mice were originally obtained from the laboratory of R. J. Lefkowitz (Duke University Medical Center, Durham, NC) and backcrossed onto a C57BL/6J background. All mice were housed on a 12-h light/12-h dark cycle in a temperature- and humidity-controlled environment and given ad libitum access to food and water. Animals were randomly assigned into treatment groups. All experiments were conducted on age-matched animals under protocols approved by the National Institute of Health Guide for the Care and Use of Laboratory Animals (IACUC of AMMS-06-2018-020). All efforts were made to minimize suffering and reduce the number of animals used.

\subsection{Reagents}

Drugs: [D-Ala2, N-MePhe4, Gly-ol]-enkephalin (DAMGO) and naloxone were purchased from Sigma-Aldrich; morphine hydrochloride was purchased from Qinghai Pharmaceutical Factory (Qinghai, China). 
Western blot antibodies: The rabbit monoclonal anti-MOR antibody (Cat\# AB1580-I, 1:500) was obtained from Millipore. The anti- $\beta$-arrestin2 antibody (LS-B6008, 1:1,000) was obtained from Life Spanbio Sciences. The Anti-FLAG M2 (F3165, 1:5,000) is purchased from Sigma-Aldrich. Anti-ABIN-1 (\#4664, 1:1,000), Anti-Myc (\#2272, 1:3,000), Anti-phos-MOR (Ser375) (\#3451, 1:1,000), Anti-ERK (\#3857, 1:1,000), and Anti-phospho-ERK (\#9154, 1:1,000),Anti-Na+ ${ }^{+} \mathrm{K}^{+}$ATPase (\#3010, 1:1,000) antibodies were purchased from Cell Signalling Technology. Anti-UbP4D1 (sc-8017, 1:1,500) and anti-IgG (sc-2025, $1 \mu \mathrm{g}$ ) antibodies were purchased from Santa Cruz Biotechnology Inc.

\subsection{Behavioural tests}

Viral constructs and Stereotaxic microinjection

AAV2/9 viruses were designed and constructed by standard methods with assistance from BrainVTA (Wuhan, China).

rAAV-CMV-ABIN-1-EGFP-WPRE-pA (AAV/PHPeB, titer: [?]3.00E + $12 \mathrm{vg} / \mathrm{ml})$ and

rAAV-U6-shRNA(ABIN-1)-CMV-EGFP-pA (AAV/PHPeB, titer: [?]5.00E+12 vg/ml) were administered via lateral ventricle injection to interfere with ABIN-1 in the brain;

rAAV-CMV-ABIN-1-EGFP-WPRE-pA (titer: [?]5.72E+12 vg/ml) and

rAAV-U6-shRNA(ABIN-1)-CMV-EGFP-pA(titer:[?]6.19E+12vg/ml) were administered via stereotaxic microinjection to interfere with ABIN-1 in the hippocampus/nucleus accumbens/anterior cingulate cortex.C57BL/6 mice were anaesthetized and placed in a stereotaxic instrument. Viruses were unilaterally injected into the right cerebral ventricle $(\mathrm{AP}=-0.6 \mathrm{~mm}, \mathrm{ML}=1.5 \mathrm{~mm}, \mathrm{DV}=2.0 \mathrm{~mm}, 5 \mu \mathrm{l}, 0.5 \mu \mathrm{l} / \mathrm{min})$ and bilaterally implanted in the anterior cingulate cortex $(\mathrm{AP}=+1.75 \mathrm{~mm}, \mathrm{ML}=0.75, \mathrm{DV}=2.65,200$ $\mathrm{nl}, 20 \mathrm{nl} / \mathrm{min}$ ), hippocampus ( $\mathrm{AP}=-2.0 \mathrm{~mm}, \mathrm{ML}=1.2 \mathrm{~mm}, \mathrm{DV}=2 \mathrm{~mm}, 400 \mathrm{nl}, 20 \mathrm{nl} / \mathrm{min}$ ) and nucleus accumbens $(\mathrm{AP}=-1.6 \mathrm{~mm}, \mathrm{ML}=0.8 \mathrm{~mm}, \mathrm{DV}=4.6 \mathrm{~mm}, 200 \mathrm{nl}, 20 \mathrm{nl} / \mathrm{min})$.

Hot plate test

The antinociceptive effects of morphine $(10 \mathrm{mg} / \mathrm{kg}$, s.c.) were assessed according to a protocol described previously(Eddy \& Leimbach, 1953) with minor modifications. Female mice were individually placed on the surface of the hot plate $\left(55 \pm 0.5^{\circ} \mathrm{C}\right)$, and the latency was recorded from the start time to the endpoint defined by jumping, licking, or shaking the hind paws. A cut-off time of 60 seconds was imposed to prevent the possibility of tissue damage. Antinociceptive data are presented as a percentage of the maximal possible effect (MPE (\%)) calculated by the following formula: \%MPE = (post drug latency -pre drug latency)/(cu $\mathrm{t}$ off time - pre drug latency) $\times 100 \%$.

Naloxone-precipitated withdrawal

Mice were exposure with morphine $(10 \mathrm{mg} / \mathrm{kg}$, s.c., twice daily) treatment for 7 days. On day 7 , withdrawal was precipitated with naloxone (i.p., $10 \mathrm{mg} / \mathrm{kg}$ ) after $2 \mathrm{~h}$ the injection of morphine. Mice were individually placed in Plexiglas boxes $(60 \mathrm{~cm} \times 20 \mathrm{~cm} \times 20 \mathrm{~cm})$ to observe the withdrawal signs(jumping) at 15 min intervals.

Conditioned place preference test (CPP)

Psychological dependence on morphine was tested using a morphine CPP test. The CPP test was performed using an unbiased, counterbalanced protocol(Wang, Zhao, Ghitza, Li \& Lu, 2008). The CPP apparatus consisted of 8 identical three-chamber polyvinyl chloride (PVC) boxes(Lu, Wu, Zhang, Ai \& Li, 2011). The two larger chambers differed by their white or black walls with a bar or grid floor texture and provided distinct contexts that were paired with morphine or saline injections. Three distinct chambers were separated by manual guillotine doors. The CPP test procedure is outlined in Fig 2.A. During the preconditioning phase (from days 1 to 3 ), male mice were given free access to all compartments of the apparatus for $15 \mathrm{~min}$. The time spent in each compartment was recorded automatically using a computer system (AniLab v3.0, AniLab Software \& Instrument Co. Ltd., Ningbo, China). Mice that showed a strong unconditioned preference (more than 540 seconds) for either compartment were excluded to maintain an unbiased population. On subsequent 
conditioning days (days 4 to 8), mice were confined to the corresponding conditioning chambers for 30 min for training. The mice were administered normal saline (N.S, $10 \mathrm{ml} / \mathrm{kg}$, i.p.) in the morning for unconditioning training and morphine $(10 \mathrm{mg} / \mathrm{kg}$, s.c.) in the afternoon during the conditioning phase. The CPP score on day 9 was defined as the time (in seconds) spent in the conditioning-paired chamber minus the time spent in the unconditioning-paired chamber during CPP testing.

\section{Locomotor activity}

Spontaneous locomotor activity was assessed using open field activity monitors

30 min before or after morphine administration on day 1. All mice were allowed to habituate to the room for $1 \mathrm{~h}$ before locomotor measurements. Activity was quantified as the horizontal distance travelled (in centimetres) over a 30-minute period.

\subsection{Tissue preparation and immunohistochemistry}

The immunofluorescence procedures were performed according to the BJP guidelines(Alexander et al., 2018)Brain tissue was obtained from morphine-injected mice with overexpression or knockdown of ABIN-1 in discrete brain regions (anterior cingulate cortex, hippocampus, and nucleus accumbens). Morphine-injected mice were deeply anaesthetized with an overdose of sodium pentobarbital $(70 \mathrm{mg} / \mathrm{kg}$, i.p.) and perfused with a $0.9 \% \mathrm{NaCl}$ solution followed by a $4 \%$ paraformaldehyde solution. The brain was removed, immersed in paraformaldehyde solution for $24 \mathrm{~h}$ followed by $30 \%$ sucrose at $4^{\circ} \mathrm{C}$ overnight and was sliced $(30 \mu \mathrm{m})$ in a freezing microtome. Immunostaining of $\beta$-arrestin2 in the hippocampus was performed using a monoclonal $\beta$-arrestin2 antibody (Life Spanbio Sciences, LS-B6008; 1:200). The immunostaining score (H-score) was calculated as follows: $\mathrm{H}-\mathrm{SCORE}=[?](\mathrm{PI} \times \mathrm{I})=($ percentage of cells with weak staining intensity $\mathrm{x} 1)+($ percentage of cells with moderate staining intensity $\mathrm{x} 2$ ) + percentage of cells with strong staining intensity $\mathrm{x} 3$ )(Azim et al., 2015).

2.5Cell culture and transfection conditions

HEK293cells were cultured in Dulbecco's modified Eagle's medium (DMEM, Thermo Fisher Scientific) containing $10 \%$ foetal bovine serum (FBS, Sigma-Aldrich). CHO cells stably expressing Flag-MOR (CHOMOR) were grown in F12 (Thermo Fisher Scientific) medium containing 10\% FBS and supplemented with G418 $(200 \mu \mathrm{g} / \mathrm{ml})$ (Thermo Fisher Scientific). CHO cells stably expressing Flag-MOR and Myc-ABIN-1 (MOR-ABIN-1-CHO) were grown in F12 medium containing 10\% FBS and supplemented with G418 (200 $\mu \mathrm{g} / \mathrm{ml})$ and hygromycin B $(200 \mu \mathrm{g} / \mathrm{ml})$. CHO cells stably expressing SNAP-MOR and EGFP- $\beta$-arrestin2 (CHO-MOR- $\beta$-arrestin2) were grown in F12 medium containing 10\% FBS and supplemented with G418 (200 $\mu \mathrm{g} / \mathrm{ml})$. Neuro-2A (N2A) cells were grown in DMEM containing 10\% FBS. All cell cultures were maintained in a humidified $5 \% \mathrm{CO}_{2}$ atmosphere at $37^{\circ} \mathrm{C}$. Transient transfections were carried out using Lipofectamine 3000 (Invitrogen) with cells grown to $65-85 \%$ confluency.

Coimmunoprecipitation

HEK293 cells in 10-cm dishes were co-transfected with the plasmid using Lipofectamine 3000 for $48 \mathrm{~h}$. Then, the cells were lysed immediately with EBC buffer (50 mM Tris- $\mathrm{HCl}[\mathrm{pH} 8.0], 120 \mathrm{mM} \mathrm{NaCl}, 0.5 \%$ NP-40, and $1 \mathrm{mM}$ EDTA) and a standard protease inhibitor mixture (Roche Applied Science) for $30 \mathrm{~min}$ on ice. The cells were centrifuged at $12,000 \mathrm{rpm}, 4^{\circ} \mathrm{C}, 20 \mathrm{~min}$. The supernatant was divided into three parts: one part was denatured by incubation in boiling water for 10 min used as input. The remaining supernatant was divided into two parts: $1 \mu \mathrm{g}$ of anti-Flag antibody was added to one part, $1 \mu \mathrm{g}$ of $\operatorname{IgG}$ with the same properties was added to the other part, shaking at 4 overnight. Next, $30 \mu \mathrm{l}$ of protein $\mathrm{G}$ magnetic beads (Thermo Fisher Scientific,\#88847) was added for $2 \mathrm{~h}$ at $4^{\circ} \mathrm{C}$. Then washed three times with EBC buffer before being eluted with $50 \mu \mathrm{l}$ of $1 \times \mathrm{SDS}$ sample buffer at $95^{\circ} \mathrm{C}$ for $5 \mathrm{~min}$.

\subsection{Phosphorylation of MOR and ERK}

MOR-CHO and MOR-ABIN-1-CHO cells were serum starved for $2 \mathrm{~h}$ prior to drug treatment. Cells were treated with DAMGO for $0 \mathrm{~min}, 5 \mathrm{~min}, 30 \mathrm{~min}, 60 \mathrm{~min}, 6 \mathrm{~h}, 24 \mathrm{~h}, 48 \mathrm{~h}$, and $72 \mathrm{~h}$ and were then lysed with lysis 
buffer (50 mM Tris-HCl [pH 7.4], $150 \mathrm{mM} \mathrm{NaCl}, 5 \mathrm{mM}$ EDTA, $10 \mathrm{mM} \mathrm{NaF}, 10 \mathrm{mM}$ disodium pyrophosphate, $1 \%$ Nonidet P-40, $0.5 \%$ sodium deoxycholate, and $0.1 \%$ SDS) with complete protease inhibitor mixture and phos-STOP phosphatase inhibitor tablets (Roche) on ice for $30 \mathrm{~min}$. The lysate was centrifuged at 12,000 $\times$ $\mathrm{g}$ for $20 \mathrm{~min}$ at $4^{\circ} \mathrm{C}$. The supernatant was separated by SDS-PAGE.

\section{$2.8 \beta$-arrestin2 ubiquitination}

pcDNA3.1myc-hisB-ABIN-1 and pCMV-Flag- $\beta$-arrestin2 were transiently transfected into HEK-293 cells. Cells were serum starved for at least $2 \mathrm{~h}$ and were then stimulated with DAMGO. Cells were solubilized in lysis buffer $(50 \mathrm{mM}$ Tris-HCl [pH 8.0], $150 \mathrm{mM} \mathrm{NaCl}, 5 \mathrm{mM}$ EDTA, $0.1 \%$ SDS, $10 \%$ glycerol, $1 \%$ Nonidet P-40, $0.5 \%$ deoxycholate, $10 \mathrm{mM}$ sodium orthovanadate, $10 \mathrm{mM} \mathrm{NaF}$, protease inhibitor mixture, and 10 mM N-ethylmaleimide). Lysates were mixed with FLAG M2 affinity beads (Sigma-Aldrich, Lot\#SLCB1079) and rotated at $4^{\circ} \mathrm{C}$ overnight. The precipitate was washed five times in RIPA buffer, and proteins were eluted with $50 \mu$ l of $2 \times$ loading buffer at $95^{\circ} \mathrm{C}$ for $10 \mathrm{~min}$.

\section{$2.9 \beta$-arrestin2 recruitment assay}

To quantify the effects of ABIN-1 on the translocation of $\beta$-arrestin2 to the membrane, pcDNA3.1myc-hisBABIN-1 was transiently transfected into $\beta$-arrestin2-GFP-MOR-CHO cells for $48 \mathrm{~h}$. Cells were then treated with $10 \mu \mathrm{M}$ DAMGO or morphine for $0,5,15$, and $30 \mathrm{~min}$. After washing in PBS, cells were fixed in $3 \%$ freshly prepared paraformaldehyde containing Hoechst $(1 \mu \mathrm{M})$ for $20 \mathrm{~min}$. Cells were then washed in PBS and imaged using an IN Cell Analyzer 2000 (20× objective; GE Healthcare Life Sciences, Pittsburgh, PA) with the following settings: exposure time of 500 milliseconds/field, excitation wavelength of $475 \mathrm{~nm}$, and emission wavelength of $535 \mathrm{~nm}$ for GFP. Images were analysed using the granularity analysis module.

\subsection{Internalization of MOR}

To further evaluate the internalization of MOR, the plasma membrane protein fraction of both cells and brain tissue was separated by a plasma membrane protein isolation kit (Invent Biotechnologies). Cells or tissue in buffer A was placed on ice for 5-10 min and was then centrifuged at 16,000 $\times \mathrm{g}$ for 30 seconds. The supernatant was discarded, and the pellet was resuspended by vigorously vertexing for 10 seconds. The resulting suspension was centrifuged at $700 \times \mathrm{g}$ for $1 \mathrm{~min}$. The supernatant was transferred to a fresh $1.5 \mathrm{ml}$ microcentrifuge tube and centrifuged at 4 for $30 \mathrm{~min}$ at 16,000 $\mathrm{x}$ g. The supernatant was removed and the pellet (the total membrane protein fraction including organelles and plasma membranes) was retained. The total membrane protein fraction was resuspended in $200 \mu \mathrm{l}$ of buffer B by repeatedly pipetting up and down or vertexing. The resulting suspension was centrifuged at 7,800 $\times \mathrm{g}$ for $5 \mathrm{~min}$ at 4 and the supernatant was removed. Ice-cold PBS $(1.6 \mathrm{ml})$ was added and centrifuged at $4 \mathrm{degC}$ and 16,000 $\mathrm{x} \mathrm{g}$ for $30 \mathrm{~min}$. The resulting pellet contained membrane proteins, which were resolved by SDS-PAGE. The supernatant was discarded, and the pellet (isolated plasma membrane proteins) was retained.

\subsection{Confocal immunofluorescence}

HEK293 cells were seeded into 35-mm cell culture dishes with coverslips attached. The dishes were coated with $0.01 \%$ poly-L-ornithine solution for $2 \mathrm{~h}$ at $37 \mathrm{degC}$ and were then washed twice with warm PBS before cell seeding. HEK293 cells were transfected with pcDNA3.1myc-hisB-ABIN-1 and pEGFP-N3- $\beta$-arrestin2 using Lipofectamine 3000 as indicated above. Immunocytochemistry was carried out as previously described(Zhou et al., 2015). Anti-myc (Abcam, 1:200) and rhodamine-conjugated Affinipure goat anti-mouse IgG antibody (1:100; Santa Cruz) were used to determine the localization of ABIN-1. Cells were then fixed with $4 \%$ paraformaldehyde for $30 \mathrm{~min}$, after which the fixed solution was aspirated and washed 3 times with PBS for 3 min each time. The cells were added to $0.5 \%$ Triton X-100 in PBS for 20 min at room temperature and washed three times in $1 \times \mathrm{PBS}$. Then, the cells were blocked for $30 \mathrm{~min}$ (1\% BSA/PBS), washed with PBST, incubated with the primary anti-myc antibody at $4^{\circ} \mathrm{C}$ overnight, and washed three times in $1 \times$ PBST. Finally, the cells were incubated with the secondary antibody for $1 \mathrm{~h}$ at room temperature and washed. The cells were treated with DAPI Fluoromount-GTM (Vector) for $5 \mathrm{~min}$ at room temperature in the dark.

\subsection{Statistical analysis}


The data and statistical analysis comply with the recommendations of British Journal of Pharmacology on experimental design and analysis in Pharmacology(Curtis et al., 2018). Statistical analyses are indicated in the figure legends and were performed using GraphPad Prism 8.0 software (GraphPad Software Inc., La Jolla, CA, USA). The results are expressed as the means \pm S.E.Ms; number of independent values(n). Samples subjected to statistical analysis were from at least 5 animals per group $(\mathrm{n}=5)$. The hotplate test results of ABIN-1 interfered in whole brain or hippocampus or anterior cingulate cortex or cell assay were analysed using two-way analysis of variance (ANOVA) followed by the Bonferroni post hoc test. The hotplate test results of ABIN-1 interfered in nucleus accumbens /naloxone-precipitated withdrawal / tissue assay results were analysis using unpaired student's t test. The criterion for statistical significance was set at $P<0.05$ for each method used.

\subsection{Nomenclature of targets and ligands}

Key protein targets and ligands in this article are hyperlinked tocorrespondingentriesinhttp://www.guidetopharmacology.org,the common portal for data from the IUPHAR/BPS Guide to PHARMACOLOGY(Harding et al., 2018), and are permanently archived in the Concise Guide to PHARMACOLOGY 2017/18(Alexander et al., 2017).

\section{Result $\mathrm{s}$}

\subsection{ABIN-1 attenuates morphine tolerance and dependence}

To investigate the role of ABIN-1 in morphine tolerance and dependence, we generated a blood-brain barriercrossing AAV to interfere with ABIN-1 in the mouse brain(Jackson, Dayton, Deverman \& Klein, 2016). The analgesic effects of morphine were assessed by hotplate test in mice injected of AAV-vector (control), AAVABIN-1 or AAV-shRNA-ABIN-1(Fig 1.A). Immunoblotting and immunofluorescence (Fig 1.B-C, Suppl 1. A) results showed that ABIN-1 expression was upregulated in the CNS(Rashnonejad et al., 2019),such as hippocampus (Hipp) or nucleus accumbens (NAc). The nociceptive latencies and locomotor behaviour of the mice were similar between ABIN-1 overexpression (or downregulation) and control group before morphine treatment (Suppl 1.B-E). The (\%) MPE differed between control and ABIN-1 group mice after morphine treatment. While Fig 1.D showed that the (\%) MPE decreased gradually after 7 consecutive days of morphine treatment $(10 \mathrm{mg} / \mathrm{kg}$, s.c.), the (\%) MPE in the ABIN-1 overexpression group was much higher than control group following chronic morphine exposure from days 1-5. And the (\%) MPE in the sh-ABIN-1 group was much lower than control group following chronic morphine exposure from days 1-5 (Fig 1. E).Together, ABIN-1 could decrease morphine tolerance.

To further investigate the effect of ABIN-1 on morphine dependence, we observed morphine withdrawal responses induced by repeated morphine exposure with subsequent naloxone administration $(10 \mathrm{mg} / \mathrm{kg}$, s.c.). Mice overexpressing ABIN-1 showed a decrease in naloxone-precipitated jumping behaviour, a withdrawal sign related to physical dependence induced by morphine (Fig 1.F). In contrast, knockdown of ABIN-1 increased mouse jumping behaviour induced by naloxone (Fig 1.G). Next, we investigated whether ABIN-1 alters vulnerability to opioid abuse. According to the CPP test procedure outlined in Fig 2.A, there is no difference between ABIN-1 overexpression or knockdown group and control group on the baseline CPP score. After 5 days of conditioned training, morphine-treated mice showed a preference for the morphine-paired compartment, but mice overexpressing ABIN-1 were less likely to prefer the box with persistent morphine injection (Fig 2.B). Consistent with the above result, knockdown of ABIN-1 in brain enhanced morphine dependence (Fig 2.C). Taken together, these results indicated that ABIN-1 in brain attenuated morphine tolerance and dependence.

\subsection{ABIN-1 in the hippocampus or in the nucleus accumbens attenuate morphine tolerance}

ABIN-1 is highly expressed in brain regions of morphine-tolerant mice, such as the cortex, hippocampus, and nucleus accumbens(Zhou et al., 2015); MOR is also highly expressed in these brain regions. To examine the effects of ABIN-1 in these regions on morphine tolerance, ABIN-1 expression was regulated separately in the hippocampus, nucleus accumbens or anterior cingulate cortex via stereotactic injection of AAV-ABIN-1 
or AAV-shABIN-1. Robust and wide-scale expression of ABIN-1 was achieved, as evidenced by the efficient labelling of neurons in the cortex, hippocampus, and nucleus accumbens, and we confirmed the efficiency of AAV transduction using western blotting (Fig 3.A-D; Supp 2.A-B). Morphine tolerance and naloxoneinduced withdrawal were assessed as described above. From days 1 to 7 , nociceptive latencies of mice with ABIN-1 overexpression or knockdown in the hippocampus or anterior cingulate cortex were the same as those of control mice before morphine treatment (Suppl 1.F-G, Suppl 2.C). However, the nociceptive latencies were higher in the mice of nucleus accumbens overexpressing ABIN-1 than control group and were lower for mice with knockdown of ABIN-1 in the nucleus accumbens than control mice prior to morphine addition (Suppl 1.H-I). Compared with control, the (\%) MPE was significantly increased in the mice of hippocampus overexpressing ABIN-1 from day 1 to day 3 after continuous administration of morphine. Naloxone-precipitated jumping behaviour was decreased by ABIN-1 overexpression in the mouse hippocampus (Fig 3. E).Similarly, ABIN-1 upregulation in the nucleus accumbens significantly increased the MPE (\%) induced by morphine and ameliorated the withdrawal syndrome (Fig 3.G). We further downregulated ABIN-1 in the hippocampus or nucleus accumbens and found that ABIN-1 knockdown had the same effect on morphine analgesia (Fig 3.F-H) and the withdrawal syndrome (Fig 3.F-H). But downregulation of ABIN-1 in the anterior cingulate cortex had no effect on morphine tolerance (Suppl 2.E-F). These findings indicated that ABIN-1 in the hippocampus or nucleus accumbens could decrease morphine tolerance.

\subsection{ABIN-1 inhibits the phosphorylation of MOR and ERK}

The interaction between ABIN-1 and MOR was confirmed in our previous work and $\beta$-arrestin signalling pathway of MOR plays an important role in the regulation of MOR signalling and trafficking(Shenoy \& Lefkowitz, 2003). MOR phosphorylation is a well-characterized prerequisite for activation of the $\beta$-arrestin2dependent pathway(Kliewer et al., 2019). Therefore, we first evaluated whether ABIN-1 could affect MOR phosphorylation. MOR phosphorylation at Ser375(Schulz S, 2004) was performed in a time-dependent manner with DAMGO $(10 \mu \mathrm{M})$ at $0 \mathrm{~min}, 5 \mathrm{~min}, 30 \mathrm{~min}, 60 \mathrm{~min}, 24 \mathrm{~h}, 48 \mathrm{~h}$, and $72 \mathrm{~h}$ (Fig 4.A-D). In MOR-CHO cells, MOR phosphorylation peaked at $5 \mathrm{~min}$ and decreased after DAMGO stimulation. And phosphorylation of MOR decreased significantly in MOR-ABIN-1-CHO cells after DAMGO stimulation at 5,30,60 min (Fig 4.A-B). Furthermore, ABIN-1 overexpression also significantly reduced MOR phosphorylation after 24, 48, and $72 \mathrm{~h}$ of DAMGO treatment (Fig 4.C-D). Earlier studies showed that ERK activity is related to the activation of $\beta$-arrestin pathway (DeWire, Ahn, Lefkowitz \& Shenoy, 2007). We speculated that ABIN-1 inhibited ERK phosphorylation like a decrease in MOR phosphorylation. As the figure showed, ERK phosphorylation peaked at $5 \mathrm{~min}$ after DAMGO treatment and decreased following DAMGO $(10 \mu \mathrm{M})$ exposure (Fig 4.A-B). The phosphorylation of ERK in MOR-ABIN-1-CHO cells was decreased obviously compared with MOR-CHO cells under acute or chronic agonist stimulation. (Fig 4.C-D). These results suggested that ABIN-1 attenuated DAMGO-induced MOR phosphorylation and ERK activation.

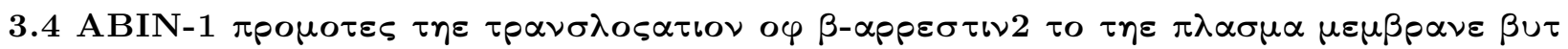

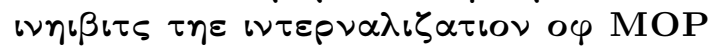

MOR phosphorylation results in recruitment of $\beta$-arrestin2 to the plasma membrane, which triggers internalization of MOR. Inhibition of MOR phosphorylation may cause a decrease in $\beta$-arrestin2 recruitment. Using high-content screening (HCS) analysis, we detected the translocation of $\beta$-arrestin 2 to the plasma membrane in $\beta$-arrestin2-EGFP-MOR-CHO cells transfected with pcDNA3.1myc-hisB-ABIN-1 or vector (Fig 5.A). Surprisingly, ABIN-1 promoted rapid translocation of $\beta$-arrestin2 to the plasma membrane after DAMGO (10 $\mu \mathrm{M})$ or Morphine $(10 \mu \mathrm{M})$ at $0,5,15$, and 30 min (Fig 5.B). Then, we evaluated the influence of ABIN-1on MOR endocytosis. Cell membrane-localized MOR was extracted from the MOR-CHO and MOR-ABIN-1 CHO cells. ABIN-1 overexpression significantly increased the amount of MOR on the cell membrane compared with control cells after DAMGO $(10 \mu \mathrm{M})$ treatment for $30 \mathrm{~min}$ or $72 \mathrm{~h}(P<0.01$, Fig $5 . \mathrm{C})$. In addition, the membrane-localized MOR was also obviously increased in the mice of ABIN-1 overexpressing compared with that in control group after chronic morphine treatment (Fig 5.D). These findings indicated that ABIN-1 promoted translocation of $\beta$-arrestin2 to the plasma membrane, but ABIN-1 attenuated endocytosis of MOR after DAMGO exposure. 


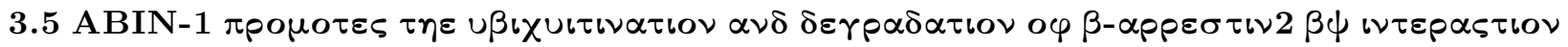 $\omega \iota \tau \eta \beta-\alpha \rho p \varepsilon \sigma \tau \iota \nu 2$}

$\beta$-arrestin2 is regard as a regulator of MOR: MOR phosphorylation recruits $\beta$-arrestin2 to induce MOR endocytosis(Lovell et al., 2015). Enhancement of $\beta$-arrestin2 recruitment by ABIN-1 occurred in the absence of opioids, suggesting a relationship between ABIN-1 and $\beta$-arrestin2. To confirm the interaction, we first detected the colocalization of ABIN-1 and $\beta$-arrestin2 and found a strong correlation in the cell membrane and cytoplasm (Pearson's R value: 0.74) (Fig 6.A). We further tested the interaction of ABIN-1 and $\beta$-arrestin2 by coimmunoprecipitation in HEK293 cells transfected with pcDNA3.1-myc-hisB-ABIN-1 (pcDNA3.1-myc-his $\mathrm{B}$ as control) and pCMV-Flag- $\beta$-arrestin2 (pCMV-Flag as control). Flag- $\beta$-arrestin2 was immunoprecipitated from lysates using an anti-Flag antibody. We found that ABIN-1 interacted with $\beta$-arrestin 2 and this interaction was augmented by DAMGO $(10 \mu \mathrm{M})$ pretreatment(Fig 6.B).ABIN-1 is well known as a ubiquitin-binding adaptor protein.(Heyninck, Kreike \& Beyaert, 2003). To determine the role of the ABIN-1 on $\beta$-arrestin2, we transfected pcDNA3.1-myc-hisB-ABIN-1, pCMV-Flag- $\beta$-arrestin2 and pcDNA3.1-myc-hisB-MOR into HEK293 cells and subsequently monitored $\beta$-arrestin2 ubiquitination. Ubiquitination of $\beta$-arrestin2 was significantly enhanced by ABIN-1 overexpression with or without DAMGO $(10 \mu \mathrm{M})$ treatment $(P<0.05)$ (Fig 6.C). Ubiquitin was originally identified as a tag for protein degradation. As expected, ABIN-1 reduced the expression of $\beta$-arrestin2 with or without DAMGO addition in N2A cells (Fig 6.D). Consistent with the results in cells, ABIN-1 also decreased the expression of $\beta$-arrestin2 in brain (hippocampus) tissue from morphine-tolerant mice after ABIN-1 overexpression. (Fig 6.E). These findings illustrated that ABIN-1 promoted $\beta$-arrestin2 ubiquitination, resulting in $\beta$-arrestin2 degradation through the interaction.

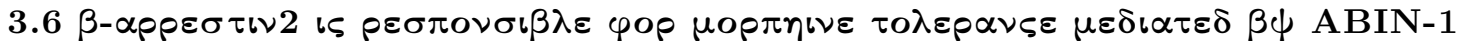

Attenuation of morphine tolerance in $\beta$-arrestin2-KO mice (ARRB2-/-) is well documented(Bohn, Lefkowitz \& Caron, 2002). Considering the suppressed expression of $\beta$-arrestin2 through its interaction with ABIN-1, ABIN-1 may target $\beta$-arrestin2 to affect morphine tolerance. ABIN-1 was upregulated or downregulated in $\mathrm{ARRB2}^{-/-}$mice by injection (i.c.v.) of AAV-ABIN-1 or AAV-shRNA-ABIN-1 respectively. The nociceptive latencies and locomotor behaviour of $\mathrm{ARRB}^{-/-}$mice were similar between ABIN-1(overexpression or knockdown) group and control group before morphine treatment (Fig 7.A-B). In ARRB2-/-mice with ABIN-1 overexpression or knockdown, the (\%) MPE was as the same as control mice from day 1 to day 7 after morphine addition $(10 \mathrm{mg} / \mathrm{kg}$ ) (Fig 7.). The behavioural results suggested that the ABIN-1-mediated attenuation of morphine tolerance was abolished in ARRB2 $2^{--}$mice, indicating that the regulation of $\beta$-arrestin2 by ABIN-1 is functionally important for morphine tolerance.

\section{Discussion}

Our recent study showed that ABIN-1 plays a critical role in the CNS and in behavioural conditioning driven by morphine. ABIN-1, which is strongly expressed in human peripheral blood lymphocytes, spleen and skeletal muscle, is essential for preventing immune system disease(Nanda et al., 2011), but its role in the CNS has rarely been reported. Using chemical genetics technique, we observed that ABIN-1 in brain attenuated morphine tolerance and dependence. The effect of ABIN-1 on morphine tolerance was inhibited in $\beta$-arrestin2-deficient mice. This behavioural effect of ABIN-1 on morphine tolerance was associated with $\beta$-arrestin signalling via MOR. Indeed, our findings established ABIN-1 as a regulator of morphine-mediated tolerance, providing keen insights into its functions.

Pain is transmitted from the spinal cord into the brain(Liu et al., 2018). Some classic pain theories, are based on research on the spinal cord, but the regulation of pain in the brain has been ignored. Therefore, in the first part of our study, we focused on the effect of ABIN-1 in brain on morphine analgesia. Using hot plate and $\mathrm{CPP}$ tests, morphine tolerance and dependence were attenuated by upregulation of ABIN-1 in brain (Fig $1,2)$. Other studies have shown that several brain regions, including the cingulate cortex, hippocampus, and nucleus accumbens(Eitan S 2003), contribute to morphine tolerance and withdrawal signs(Koob \& Volkow, 2010). In the present study, overexpression of ABIN-1 in mice hippocampus or nucleus accumbens was sufficient to reduce morphine tolerance and physical dependence after chronic morphine addition (Fig 3). The 
role of the hippocampus in the formation of memory and cognition is well known. Early studies showed that partial hippocampectomy could treat chronic pain in humans(Jarrard \& Lewis, 1967), demonstrating that the hippocampus is involved in nociception and morphine-induced antinociception(Hashemi, Karami, Zarrindast \& Sahebgharani, 2010; Soleimannejad, Semnanian, Fathollahi \& Naghdi, 2006). Several studies support the idea that the hippocampal region participates in morphine-induced antinociception(Hashemi, Karami, Zarrindast \& Sahebgharani, 2010) and that the ventral hippocampal CA1-infralimbic cortex modulates the progression of pain in rats with peripheral inflammation(Ma et al., 2019). In addition, withdrawal behaviour induced by opioid antagonists implies anxiety and stress, which are related to emotionality. The hippocampus is a key region that controls this emotionality(Bremner J D 2000). MOR and ABIN-1 are widely coexpressed in the hippocampus; thereby ABIN-1 in the mouse hippocampus participates in morphine-induced antinociception and withdrawal behaviour.

Similarly, ABIN-1 in the nucleus accumbens is also involved in the regulation of morphine tolerance (Fig 3). Interestingly, we found a striking difference in the regulation pattern of the nociceptive latency: knockdown of ABIN-1 in the nucleus accumbens specifically reduced the nociceptive latency and leading to hyperalgesia before morphine treatment in mice (Fig 3). The nucleus accumbens is another brain region thought to be critical in mediating opioid reward and addiction(Sadat-Shirazi et al., 2019; Xi, Wu, Stein \& Li, 2004) and to play a role in co-morbid hyperalgesia under opioid exposure(Zhang et al., 2019). MOR and ABIN-1 are expressed in the nucleus accumbens, and the expression of MORs is modulated by morphine treatment(Johnson, Chieng, Napier \& Connor, 2006). ABINs have been described as ubiquitinated proteins that negatively regulate nuclear factor (NF- $x \mathrm{~B})$ activation(Verstrepen, Carpentier, Verhelst \& Beyaert, 2009). Knockdown of ABIN-1 promotes the activation of NF- $\varkappa$ B, which may remodel and change the shape of nucleus accumbens neurons(Ang E 2001) to affect the basal nociception latency(Taylor, Becker, Schweinhardt \& Cahill, 2016). Indeed, our findings suggest that ABIN-1 has clinical utility as a morphine sensitizer. Further study is needed to understand the function of ABIN-1 in brain neurons.

While the sustained activation of $\mathrm{G}$ protein signalling is understood as the classical transduction of morphine analgesic, the tolerance mechanism remains unclear(Williams et al., 2013). $\beta$-arrestin signalling exerts a vital function on MOR desensitization and can compete with G-protein signalling(Kovacs, Hara, Davenport, Kim \& Lefkowitz, 2009). Our data showed that ABIN-1 decreased MOR phosphorylation and internalization under treatment with agonists (Fig 4-5), presumably reflecting downregulation of the $\beta$-arrestin pathway. Whereas ABIN-1 promoted the accumulation of $\beta$-arrestin2 on the plasma membrane (Fig 5). Theoretically, MOR phosphorylation is more likely to recruit $\beta$-arrestin- 2 , and reduction of MOR phosphorylation may prevent the translocation of $\beta$-arrestin2 to the plasma membrane. Seeking for the mechanism, we found $\beta$-arrestin2 interacted with ABIN-1, and ABIN-1 also bound to MOR, which might promote $\beta$-arrestin2 to the plasma membrane (Fig 6). Receptor phosphorylation is required for sustained interaction with GRKs under agonist activation(Inagaki et al., 2015). Two lines of evidence may explain why ABIN-1 decreased agonist-induced MOR phosphorylation. First, our previous work has proved that ABIN-1 could interact with MOR-C-terminus. The C-terminus of MOR contains an abundance phosphorylation sites, and ABIN-1 may compete with GRKs to decrease MOR phosphorylation. Second, we found that ABIN-1 could also bind to $\beta$-arrestin2. ABIN-1, $\beta$-arrestin2 and MOR functionally interacted with each other to produce strong steric hindrance; thus, phosphorylation of MOR by GRKs was decreased. The magnitude and duration of morphine-induced analgesia was affected in the mice MOR phosphorylation deficiency (Kliewer et al., 2019), that indicated ABIN-1 reduced MOR phosphorylation to involve in morphine tolerance. Modulation of MOR phosphorylation influences the association between $\beta$-arrestin and MOR, dissociation of the $\beta$ arrestin-receptor complex results in impairment of MOR endocytosis. Our findings showed that ABIN-1 decreased MOR internalization (Fig 5). Classical theory states that a reduction in the amount of MOR on the plasma membrane is related to morphine tolerance. Briefly, ABIN-1 delayed the development of morphine tolerance by negatively regulating the $\beta$-arrestin signalling pathway by reducing the phosphorylation and internalization of MOR.

ABINs are considered as ubiquitinated adaptors (Zhou, 2011). We found a novel consequence of the ABIN$1-\beta$-arrestin-2 complex, which could promote the ubiquitination and degradation of $\beta$-arrestin-2 (Fig 6). 
$\beta$-Arrestin2, as a multifunctional adaptor, mediates MOR trafficking and transduction to regulate morphine tolerance. Downregulation of $\beta$-arrestin2 plays a vital role in MOR desensitization(Jean-Charles, Freedman \& Shenoy, 2016). For G protein-dependent signalling of MOR, $\beta$-arrestin2 increases cAMP degradation by recruiting and scaffolding cellular phosphodiesterases (PDEs), which could diminish the accumulation of cAMP. Thus, downregulation of $\beta$-arrestin2 results in an increase in cAMP; moreover, we previously found that ABIN-1 increased the accumulation of cAMP(Zhou, 2017). For $\beta$-arrestin-dependent signalling, morphine actives MOR and recruits only $\beta$-arrestin2(Groer, Schmid, Jaeger \& Bohn, 2011). The membrane proteins are often ubiquitinated during arrestin-mediated internalization(Mittal \& McMahon, 2009). In CNS neurons, neurokinin 1 receptors mediate the sequestration of arrestins on endosomal membranes and reduce opioid-induced desensitization(Yu, Arttamangkul, Evans, Williams \& von Zastrow, 2009). MOR desensitization and morphine tolerance are reversed in $\beta$-arrestin2-knockout mice(Bohn, Lefkowitz \& Caron, 2002). In $\beta$-arrestin2-knockout mice, the severity of antagonist-precipitated withdrawal signs is attenuated after chronic morphine treatment, supporting the idea that $\beta$ arrestin2 plays a role in morphine tolerance and dependence(Raehal \& Bohn, 2011). Due to the downregulating of MOR phosphorylation and degradation of $\beta$-arrestin2 induced by ABIN-1 overexpression (Fig 4-6), the MOR on the plasma membrane was increased in both morphine-tolerant mice brains and cell lines. Therefore, the lack of MOR desensitization resulting from the decrease in endocytosis altered the normal signalling response to opioids. As a result, ABIN-1 had no effect on morphine tolerance in $\beta$-arrestin2-knockout mice (Fig 7). Collectively, ABIN-1 promotes $\beta$-arrestin2 degradation to attenuate morphine tolerance.

$\beta$-arrestins act as scaffold-specific components of the mitogen-activated protein kinase (MAPK) cascade(Luttrell et al., 2001). Inhibition of the MAPK pathway blocks desensitization of MOR signalling as well as the loss of receptor expression due to internalization(Polakiewicz, 1998). ERK1 and 2, members of the MAPK family, are crucial regulators of MOR signal transduction(Zheng H 2007). In our present study, phosphorylation of ERK was inhibited by ABIN-1 under both acute and chronic opioid treatment (Fig 4). As a downstream molecule in both the G protein- and $\beta$-arrestin-dependent pathways(Muller \& Unterwald, 2004), ABIN-1 regulates ERK activation by negatively affecting both the $G$ protein and $\beta$-arrestin signalling pathways. And ABIN-1 interacted with ERK to attenuate ERK signalling(Zhang, 2002), which might alter MOR signal transduction. Additionally, there was a study showed that A20 upregulation attenuated morphine tolerance by inhibiting the activation of $\mathrm{NF}-\varkappa \mathrm{B}$ and ABIN-1 through the interaction of AHD1 with A20(Huang et al., 2019). Therefore, ABIN-1 and A20 may work together to participate in mediating morphine tolerance.

In summary, the ABIN-1-MOR- $\beta$-arrestin2 complex functionally diminished MOR phosphorylation and promoted $\beta$-arrestin2 degradation, which decreased MOR internalization. Thus, ABIN-1 overexpression in mouse brain alleviated morphine tolerance and dependence. The therapeutic effect of ABIN-1 on morphine tolerance was blocked in $\beta$-arrestin2-knockout mice. Overall, ABIN-1 may be a target for alleviating morphine tolerance.

\section{Acknowledgments}

This work was supported by grants from the National Natural Science Foundation of China (81473194).

\section{Authorship Contributions}

designing research studies: Zhou, Su, Gong

conducting experiments: Zhang, Lu

analyzing data: Zhang, Zhou.

providing reagents:Zhou, Su.

writing the manuscript: Zhang, Zhou, Su.

\section{Conflict of interest}


The authors declare no conflicts of interest.

\section{Declaration of transparency and scientific rigour}

This Declaration acknowledges that this paper adheres to the principles for transparent reporting and scientific rigour of preclinical research as stated in the BJP guidelines for Design \& Analysis, and Animal Experimentation, and as recommended by funding agencies, publishers and other organizations engaged with supporting research.

\section{References}

Al-Hasani R, \& Bruchas MR (2011). Molecular mechanisms of opioid receptor-dependent signaling and behavior. Anesthesiology 115:1363-1381.

Alexander SP, Christopoulos A, Davenport AP, Kelly E, Marrion NV, Peters JA, et al. (2017). THE CONCISE GUIDE TO PHARMACOLOGY 2017/18: G protein-coupled receptors. British journal of pharmacology 174 Suppl 1: S17-s129.

Alexander SPH, Roberts RE, Broughton BRS, Sobey CG, George CH, Stanford SC, et al. (2018). Goals and practicalities of immunoblotting and immunohistochemistry: A guide for submission to the British Journal of Pharmacology. British journal of pharmacology 175: 407-411.

Ang E CJ, Zagouras P , et al ( 2001). Induction of nuclear factor-kappaB in nucleus accumbens by chronic cocaine administration. Journal of Neurochemistry 79: :221-224.

Attramadal H, ., Arriza JL, Aoki C, ., Dawson TM, Codina J, ., Kwatra MM, et al. (1992). Beta-arrestin2, a novel member of the arrestin/beta-arrestin gene family. Journal of Biological Chemistry 267: 17882-17890.

Azim HA, Jr., Peccatori FA, Brohee S, Branstetter D, Loi S, Viale G, et al. (2015). RANK-ligand (RANKL) expression in young breast cancer patients and during pregnancy. Breast cancer research : BCR 17: 24.

Basso Lilian AR, Fan Churmy Yong et al. (20). TRPV1 promotes opioid analgesia during inflammation. Sci Signal 12.

Bohn LM, Lefkowitz RJ, \& Caron MG (2002). Differential mechanisms of morphine antinociceptive tolerance revealed in (beta)arrestin-2 knock-out mice. The Journal of neuroscience : the official journal of the Society for Neuroscience 22: 10494-10500.

Bremner J D NM, Anderson E R, et al. n (2000). Hippocampal Volume Reduction in Major Depression. American Journal of Psychiatry 157: :115-118.

Curtis MJ, Alexander S, Cirino G, Docherty JR, George CH, Giembycz MA, et al. (2018). Experimental design and analysis and their reporting II: updated and simplified guidance for authors and peer reviewers. British journal of pharmacology 175: 987-993.

DeWire SM, Ahn S, Lefkowitz RJ, \& Shenoy SK (2007). Beta-arrestins and cell signaling. Annu Rev Physiol 69: $483-510$.

Eddy NB, \& Leimbach D (1953). Synthetic analgesics. II. Dithienylbutenyl- and dithienylbutylamines. Journal of Pharmacology \& Experimental Therapeutics 107: 385-393.

Eitan S BCD, Saliminejad N , et al. (2003). Brain Region-Specific Mechanisms for Acute MorphineInduced Mitogen-Activated Protein Kinase Modulation and Distinct Patterns of Activation during Analgesic Tolerance and Locomotor Sensitization. Journal of Neuroscience the Official Journal of the Society for Neuroscience 23: 8360-8369.

Groer CE, Schmid CL, Jaeger AM, \& Bohn LM (2011). Agonist-directed interactions with specific betaarrestins determine mu-opioid receptor trafficking, ubiquitination, and dephosphorylation. J Biol Chem 286: 31731-31741. 
Harding SD, Sharman JL, Faccenda E, Southan C, Pawson AJ, Ireland S, et al. (2018). The IUPHAR/BPS Guide to PHARMACOLOGY in 2018: updates and expansion to encompass the new guide to IMMUNOPHARMACOLOGY. Nucleic acids research 46: D1091-d1106.

Hashemi M, Karami M, Zarrindast MR, \& Sahebgharani M (2010). Role of nitric oxide in the rat hippocampal CA1 in morphine antinociception. Brain Res 1313: 79-88.

Heyninck K, Kreike MM, \& Beyaert R (2003). Structure-function analysis of the A20-binding inhibitor of NF- $\chi \mathrm{B}$ activation, ABIN-1. FEBS Letters 536: 135-140.

Huang J, Liang X, Wang J, Kong Y, Zhang Z, Ding Z, et al. (2019). miR-873a-5p Targets A20 to Facilitate Morphine Tolerance in Mice. Front Neurosci 13: 347.

Inagaki S, Ghirlando R, Vishnivetskiy SA, Homan KT, White JF, Tesmer JJ, et al. (2015). G ProteinCoupled Receptor Kinase 2 (GRK2) and 5 (GRK5) Exhibit Selective Phosphorylation of the Neurotensin Receptor in Vitro. Biochemistry 54: 4320-4329.

Jackson KL, Dayton RD, Deverman BE, \& Klein RL (2016). Better Targeting, Better Efficiency for WideScale Neuronal Transduction with the Synapsin Promoter and AAV-PHP.B. Frontiers in molecular neuroscience 9: 116.

Jarrard LE, \& Lewis TC (1967). Effects of Hippocampal Ablation and Intertrial Interval on Acquisition and Extinction in a Complex Maze. American Journal of Psychology 80: 66-72.

Jean-Charles PY, Freedman NJ, \& Shenoy SK (2016). Chapter Nine - Cellular Roles of Beta-Arrestins as Substrates and Adaptors of Ubiquitination and Deubiquitination. Progress in molecular biology and translational science 141: 339-369.

Johnson EE, Chieng B, Napier I, \& Connor M (2006). Decreased mu-opioid receptor signalling and a reduction in calcium current density in sensory neurons from chronically morphine-treated mice. British journal of pharmacology 148: 947-955.

Kliewer A, Schmiedel F, Sianati S, Bailey A, Bateman JT, Levitt ES, et al. (2019). Phosphorylationdeficient G-protein-biased mu-opioid receptors improve analgesia and diminish tolerance but worsen opioid side effects. Nature communications 10: 367.

Koob GF, \& Volkow ND (2010). Neurocircuitry of addiction. Neuropsychopharmacology : official publication of the American College of Neuropsychopharmacology 35: 217-238.

Koshimizu TA, Honda K, Nagaoka-Uozumi S, Ichimura A, Kimura I, Nakaya M, et al. (2018). Complex formation between the vasopressin $1 \mathrm{~b}$ receptor, beta-arrestin-2, and the mu-opioid receptor underlies morphine tolerance. Nat Neurosci 21: 820-833.

Kovacs JJ, Hara MR, Davenport CL, Kim J, \& Lefkowitz RJ (2009). Arrestin development: emerging roles for beta-arrestins in developmental signaling pathways. Developmental cell 17: 443-458.

Liu Y, Latremoliere A, Li X, Zhang Z, Chen M, Wang X, et al.(2018). Touch and tactile neuropathic pain sensitivity are set by corticospinal projections. Nature 561: 547-550.

Lovell KM, Frankowski KJ, Stahl EL, Slauson SR, Yoo E, Prisinzano TE, et al. (2015). Structure-activity relationship studies of functionally selective kappa opioid receptor agonists that modulate ERK $1 / 2$ phosphorylation while preserving $\mathrm{G}$ protein over betaarrestin2 signaling bias. ACS chemical neuroscience 6: 1411-1419.

Lu GY, Wu N, Zhang ZL, Ai J, \& Li J (2011). Effects of D-cycloserine on extinction and reinstatement of morphine-induced conditioned place preference. Neurosci Lett 503: 196-199.

Luttrell LM, Roudabush FL, Choy EW, Miller WE, Field ME, Pierce KL, et al. (2001). Activation and targeting of extracellular signal-regulated kinases by beta-arrestin scaffolds. Proc Natl Acad Sci U S A 98: 
2449-2454.

Ma L, Yue L, Zhang Y, Wang Y, Han B, Cui S, et al. (2019). Spontaneous Pain Disrupts Ventral Hippocampal CA1-Infralimbic Cortex Connectivity and Modulates Pain Progression in Rats with Peripheral Inflammation. Cell Rep 29: 1579-1593.

Manglik A, Lin H, Aryal DK, McCorvy JD, Dengler D, Corder G, et al. (2016). Structure-based discovery of opioid analgesics with reduced side effects. Nature 537: 185-190.

Mittal R, \& McMahon HT (2009). Arrestins as adaptors for ubiquitination in endocytosis and sorting. EMBO Rep 10: 41-43.

Molinari P, Vezzi V, Sbraccia M, Gro C, Riitano D, Ambrosio C, et al. (2010). Morphine-like opiates selectively antagonize receptor-arrestin interactions. J Biol Chem 285: 12522-12535.

Muller DL, \& Unterwald EM (2004). In vivo regulation of extracellular signal-regulated protein kinase (ERK) and protein kinase B (Akt) phosphorylation by acute and chronic morphine. The Journal of pharmacology and experimental therapeutics 310: 774-782.

Nanda SK, Venigalla RK, Ordureau A, Patterson-Kane JC, Powell DW, Toth R, et al. (2011). Polyubiquitin binding to ABIN1 is required to prevent autoimmunity. J Exp Med 208: 1215-1228.

Polakiewicz RD, Schieferl, S. M., Dorner, L. F., Kansra, V., \& Comb, M. J. (1998). A Mitogen-activated Protein Kinase Pathway Is Required for $\mu$-Opioid Receptor Desensitization. Journal of Biological Chemistry 273: 12402-12406.

Raehal KM, \& Bohn LM (2011). The role of beta-arrestin2 in the severity of antinociceptive tolerance and physical dependence induced by different opioid pain therapeutics. Neuropharmacology 60:58-65.

Rashnonejad A, Amini Chermahini G, Gunduz C, Onay H, Aykut A, Durmaz B, et al. (2019). Fetal Gene Therapy Using a Single Injection of Recombinant AAV9 Rescued SMA Phenotype in Mice. Mol Ther 27:2123-2133.

Sadat-Shirazi MS, Neirizi NM, Matloob M, Safarzadeh M, Behrouzi M, Dehdashti AR, et al. (2019). Possible involvement of nucleus accumbens D1-like dopamine receptors in the morphine-induced condition place preference in the offspring of morphine abstinent rats. Life sciences: 116712.

Schulz S MD, Pfeiffer M, et al (2004). Morphine induces terminal $\mu$-opioid receptor desensitization by sustained phosphorylation of serine-375. The EMBO journal 23: 3282-3289.

Sharma S K KWA, Nirenberg M . (1975). Dual regulation of adenylate cyclase accounts for narcotic dependence and tolerance. Proceedings of the National Academy of Sciences, 72: 3092-3096.

Shenoy SK, \& Lefkowitz RJ (2003). Multifaceted roles of beta-arrestins in the regulation of seven-membranespanning receptor trafficking and signalling. Biochem J 375: 503-515.

Soleimannejad E, Semnanian S, Fathollahi Y, \& Naghdi N (2006). Microinjection of ritanserin into the dorsal hippocampal CA1 and dentate gyrus decrease nociceptive behavior in adult male rat. Behav Brain Res 168: 221-225.

Takenouchi O, Yoshimura H, \& Ozawa T (2018). Unique Roles of beta-Arrestin in GPCR Trafficking Revealed by Photoinducible Dimerizers. Scientific reports 8: 677.

Taylor AM, Becker S, Schweinhardt P, \& Cahill C (2016). Mesolimbic dopamine signaling in acute and chronic pain: implications for motivation, analgesia, and addiction. Pain 157: 1194-1198.

Verstrepen L, Carpentier I, Verhelst K, \& Beyaert R (2009). ABINs: A20 binding inhibitors of NF-kappa B and apoptosis signaling. Biochemical pharmacology 78: 105-114. 
Wang XY, Zhao M, Ghitza UE, Li YQ, \& Lu L (2008). Stress impairs reconsolidation of drug memory via glucocorticoid receptors in the basolateral amygdala. The Journal of neuroscience : the official journal of the Society for Neuroscience 28: 5602-5610.

Williams JT, Ingram SL, Henderson G, Chavkin C, von Zastrow M, Schulz S, et al. (2013). Regulation of mu-opioid receptors: desensitization, phosphorylation, internalization, and tolerance. Pharmacological reviews 65: 223-254.

Wu XP, She RX, Yang YP, Xing ZM, Chen HW, \& Zhang YW (2018). MicroRNA-365 alleviates morphine analgesic tolerance via the inactivation of the ERK/CREB signaling pathway by negatively targeting betaarrestin2. Journal of biomedical science 25: 10.

Xi ZX, Wu G, Stein EA, \& Li SJ (2004). Opiate tolerance by heroin self-administration: an fMRI study in rat. Magnetic resonance in medicine 52: 108-114.

Yu YJ, Arttamangkul S, Evans CJ, Williams JT, \& von Zastrow M (2009). Neurokinin 1 receptors regulate morphine-induced endocytosis and desensitization of mu-opioid receptors in CNS neurons. The Journal of neuroscience : the official journal of the Society for Neuroscience 29: 222-233.

Zhang P, Moye LS, Southey BR, Dripps I, Sweedler JV, Pradhan A, et al. (2019). Opioid-Induced Hyperalgesia Is Associated with Dysregulation of Circadian Rhythm and Adaptive Immune Pathways in the Mouse Trigeminal Ganglia and Nucleus Accumbens. Molecular neurobiology.

Zhang S, Fukushi, M., Hashimoto, S., Gao, C., Huang, L., Fukuyo, Y., ... Tsuchida (2002). A new ERK2 binding protein, Naf1, attenuates the EGF/ERK2 nuclear signaling. Biochemical and Biophysical Research Communications 297: 17-23.

Zheng H LHH, Law P Y ( 2007). $\beta$-Arrestin-Dependent $\mu$-Opioid Receptor-Activated Extracellular SignalRegulated Kinases (ERKs) Translocate to Nucleus in Contrast to G Protein-Dependent ERK Activation. Molecular Pharmacology, 73: 178-190.

Zhou J, Wu, R., High, A. A., Slaughter, C. A., Finkelstein, D., Rehg, J. E., ... Hacker, H. (2011). A20binding inhibitor of NF- B (ABIN1) controls Toll-like receptor-mediated CCAAT/enhancer-binding protein activation and protects from inflammatory disease. Proceedings of the National Academy of Sciences, 108: 998-1006.

Zhou P, Jiang J, Dong Z, Yan H, You Z, Su R, et al. (2015). The proteins interacting with C-terminal of $\mathrm{mu}$ receptor are identified by bacterial two-hybrid system from brain cDNA library in morphine-dependent rats. Life sciences 143: 156-167.

Zhou P, Jiang, J., Yan, H., Li, Y., Zhao, J., Wang, X., ... Gong, Z. (2017). ABIN-1 Negatively Regulates $\mu-$ Opioid Receptor Function. Molecular Pharmacology, 93: 36-48. 


\section{Figure1}

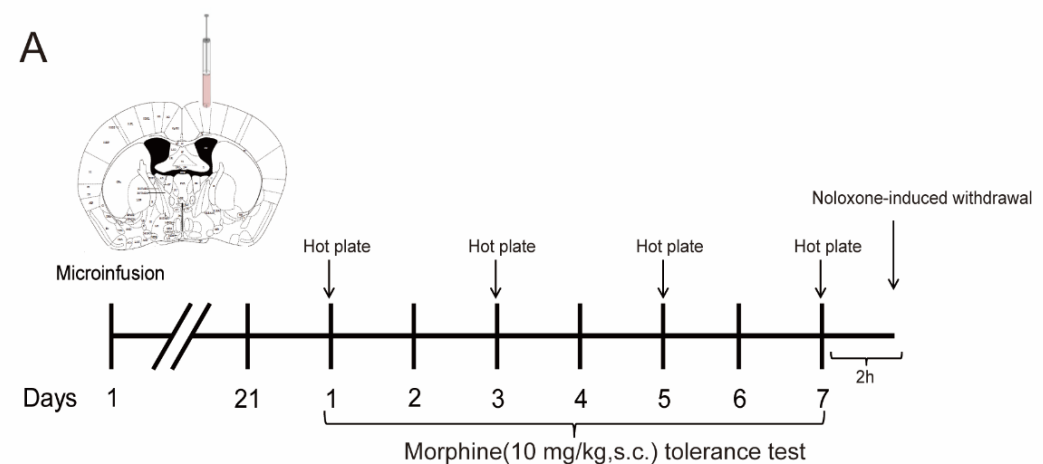

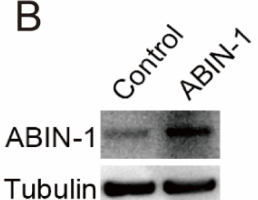

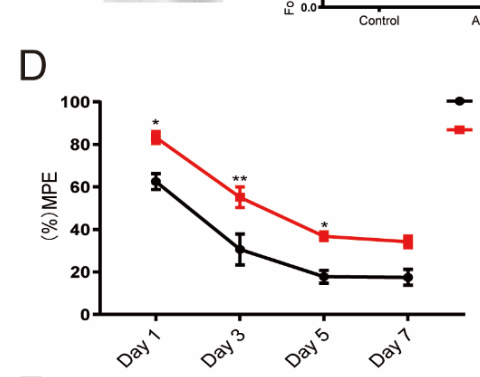

$\mathrm{F}$

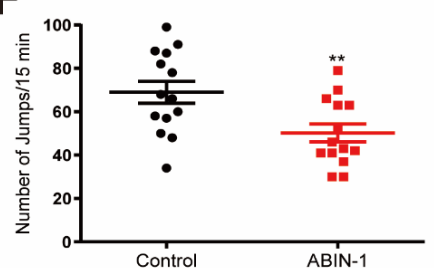

C
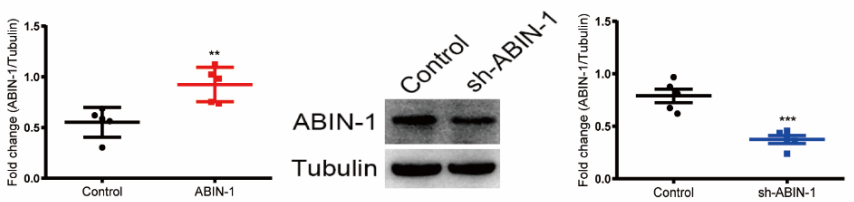

E

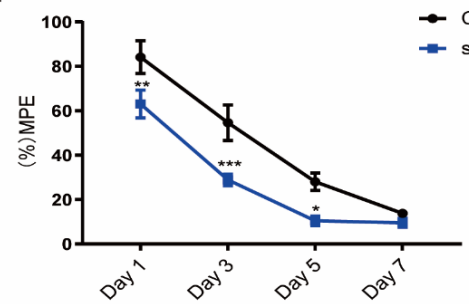

G

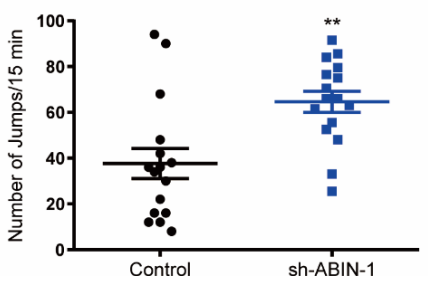

Fig. 1 ABIN-1 in the brain alleviated morphine tolerance. (A)Experimental schedule for morphineinduced tolerance and the naloxone-induced withdrawal test. (B-C) Immunoblotting analysis and quantification of ABIN-1 expression was interfered by AAV-ABIN-1(B) or AAV-shRNA-ABIN-1(C) in the mouse brain. (D-E) The (\%) MPE of analgesia induced by chronic morphine treatment was detected after ABIN1overexpression (D)or knockdown (E) in the mouse brain. (F-G) The jumping counts precipitated by naloxone after chronic morphine treatment in ABIN-1 up-regulated (E) or down-regulated (F)mice.Twoway ANOVA followed by Bonferroni post hoc test, $\mathrm{n}=8-9$; The western blot results were analysed with Student t-test, $\mathrm{n}=5$, ABIN-1 vs control, ${ }^{*} P<0.05,{ }^{*} P<0.01,{ }^{* * *} P<0.001$. The error bars indicate the means \pm S.E.Ms. 
Figure2

A

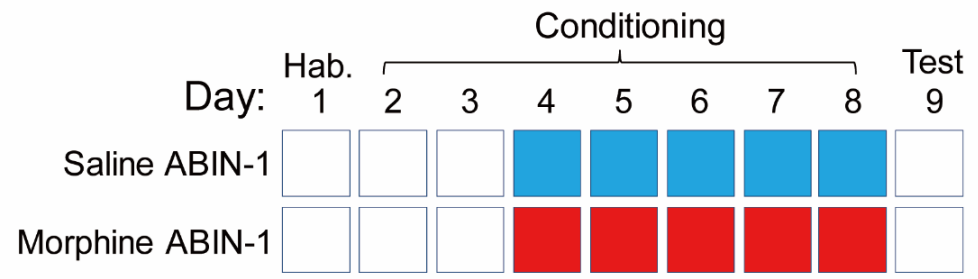

Free access to both chambers

Saline in conditioned chamber

Morphine $(10 \mathrm{mg} / \mathrm{kg})$ in conditioned chamber

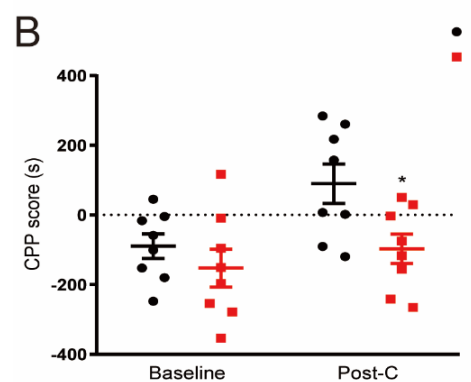

C

- $A B \mid N-1$

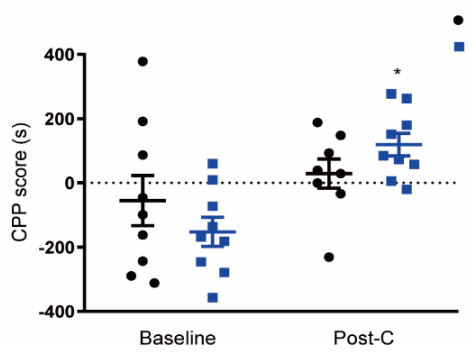

Fig. 2 ABIN-1 in the brain alleviated morphine dependence. (A)Experimental schedule for CPP test. (B-C) The results are assessed as CPP score (time spent in morphine - paired side on post-conditioning - the time on preconditioning) in the mouse brain after overexpression (B) or knockdown (C) the ABIN-1. Student's t test, ABIN-1 vs control, ${ }^{*} P<0.05 ; \mathrm{n}=8-9$. The error bars indicate the means \pm S.E.Ms. 
A
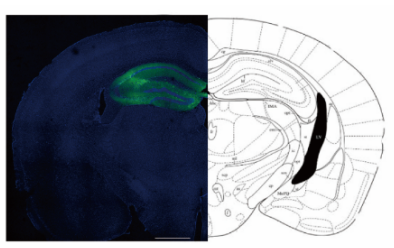

C
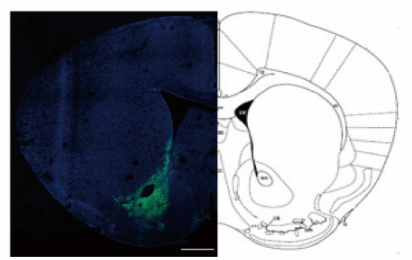

E
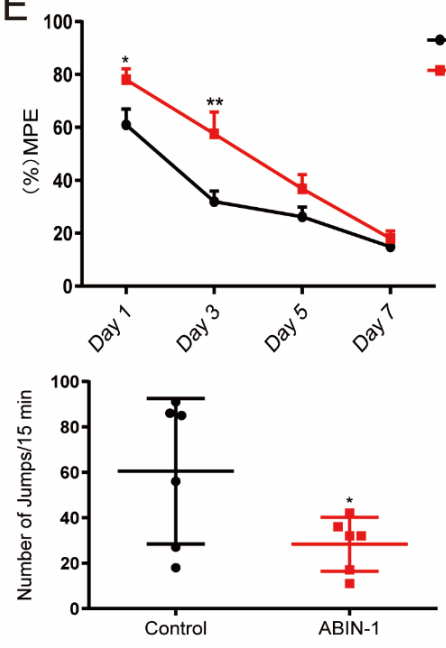

G
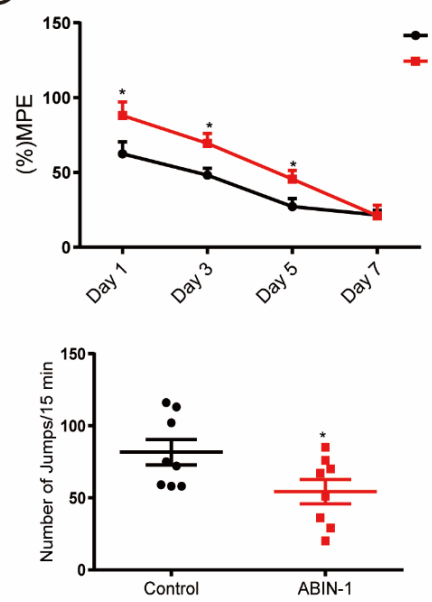

B

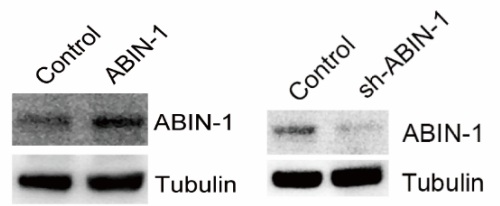

D

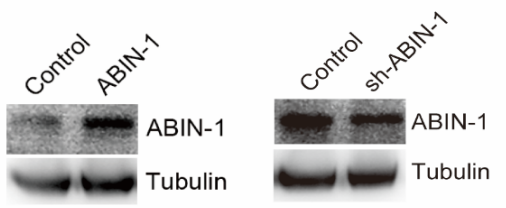

$\mathrm{F}$

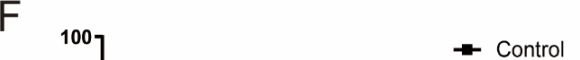

${ }^{100}$

- ABIN-1
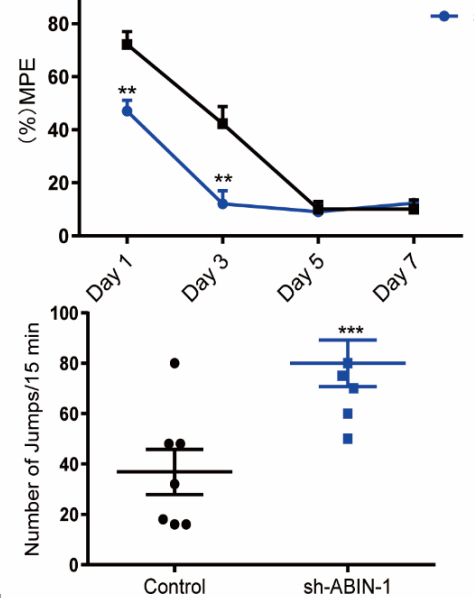

$\mathrm{H}$
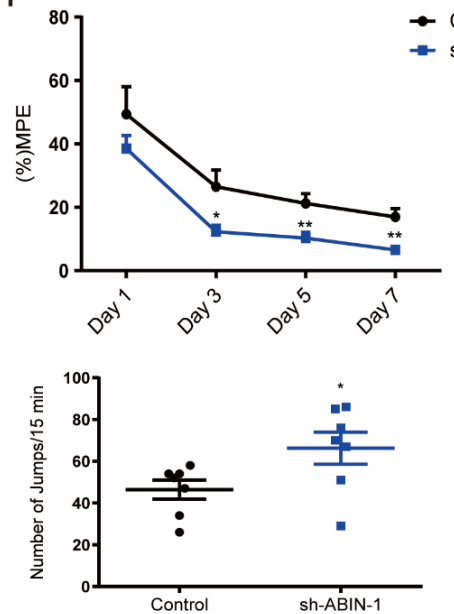

Fig. 3 ABIN-1 in the hippocampusor nucleus accumbens decreases morphine tolerance. (A,C) AAV-ABIN-1-EGFP-labelled neurons in the hippocampus(A) or in the nucleus accumbens(C) . Blue, DAPI. Scale bar, $1000 \mu \mathrm{m}$. (B) ABIN-1 expression in the hippocampus was analysed by immunoblotting after interfered by the AAV-ABIN-1(left) or AAV-shRNA-ABIN-1(right).(D) ABIN-1 expression in the nucleus accumbens was analysed by immunoblotting after interfered by the AAV-ABIN-1(left) or AAV-shRNAABIN-1(right) .(E-F) In the mouse hippocampus up-regulated (E) or down-regulated (F) ABIN-1, the 
(\%)MPE of analgesia induced by chronic morphine treatment (up) and the jumping counts precipitated by naloxone (down) was detected. (G-H) In the mouse nucleus accumbens up-regulated (G) or down-regulated (H)ABIN-1, the (\%) MPE of analgesia induced by chronic morphine treatment (up) followed by the jumping counts precipitated by naloxone (doen) was detected. The MPE (\%) was analysed with two-way ANOVA followed by Bonferroni post hoc test; The number of jumps was analysed with a Student's t test. ${ }^{*} P<0.05$, ${ }^{* *} P<0.01,{ }^{* * *} P<0.001 ; \mathrm{n}=7-8$. The error bars indicate the means \pm S.E.Ms.

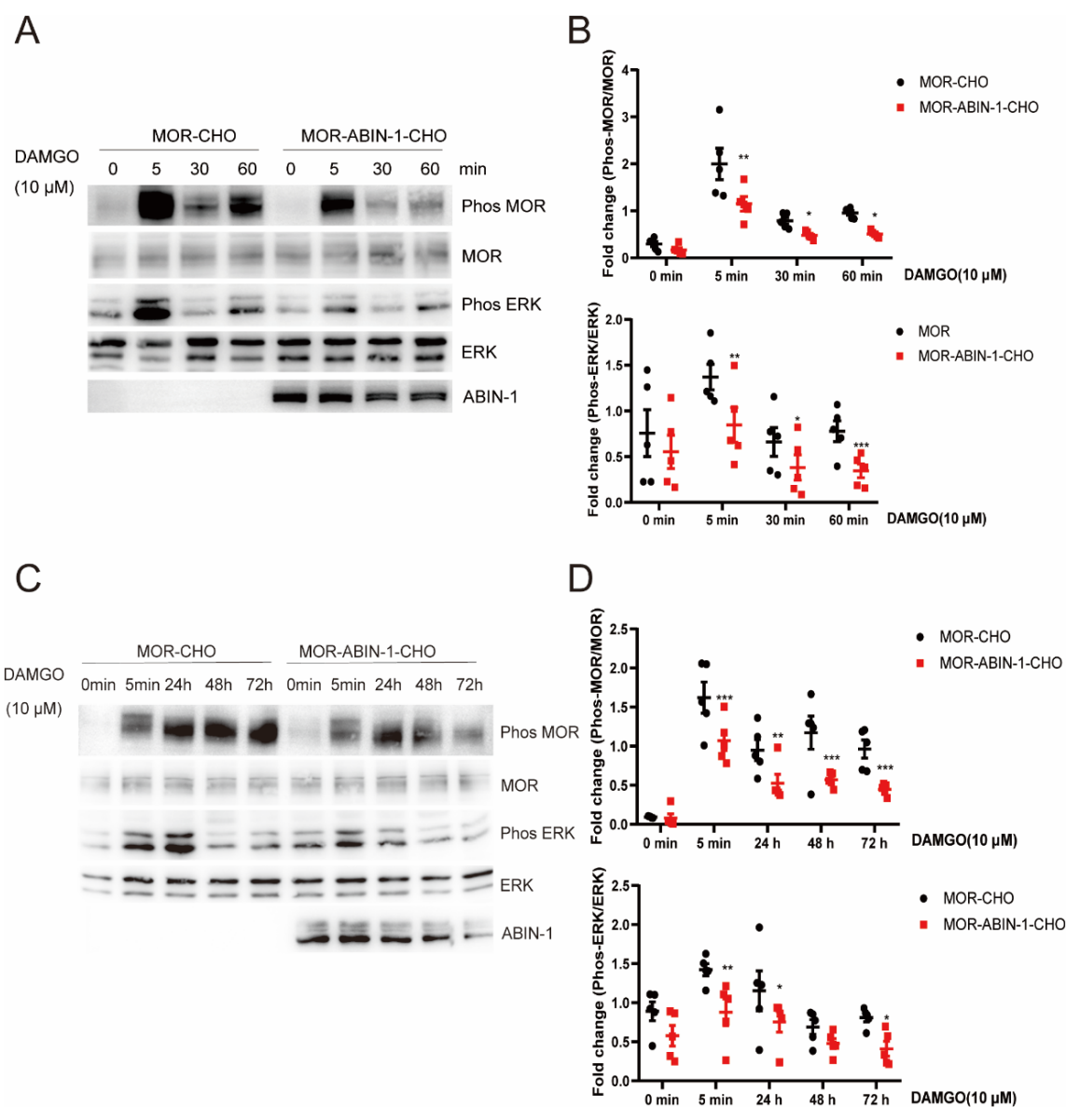

Fig. 4 ABIN-1 inhibits the phosphorylation of MOR and ERK. (A-B) Phosphorylation of MOR (phos MOR, Ser375) and ERK (phos ERK) in MOR-CHO and MOR-ABIN-1-CHO cells treated with DAMGO $(10 \mu \mathrm{M})$ for $0,5,30$, or $60 \mathrm{~min}(\mathbf{A})$ or 24,36 , or $48 \mathrm{~h}(\mathbf{B})$ was detected by immunoblotting. Two-way ANOVA followed by Bonferroni post hoc test, ABIN-1 vs control, ${ }^{*} P<0.05,{ }^{* *} P<0.01$, ${ }^{* * *} P$ $<0.001 ; \mathrm{n}=5$. The error bars indicate the means \pm S.E.Ms. 
A

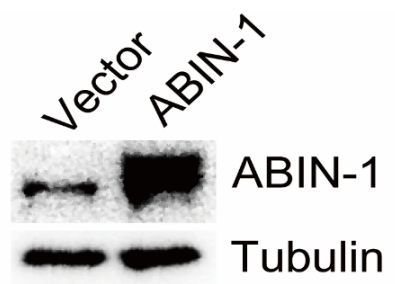

B

r.
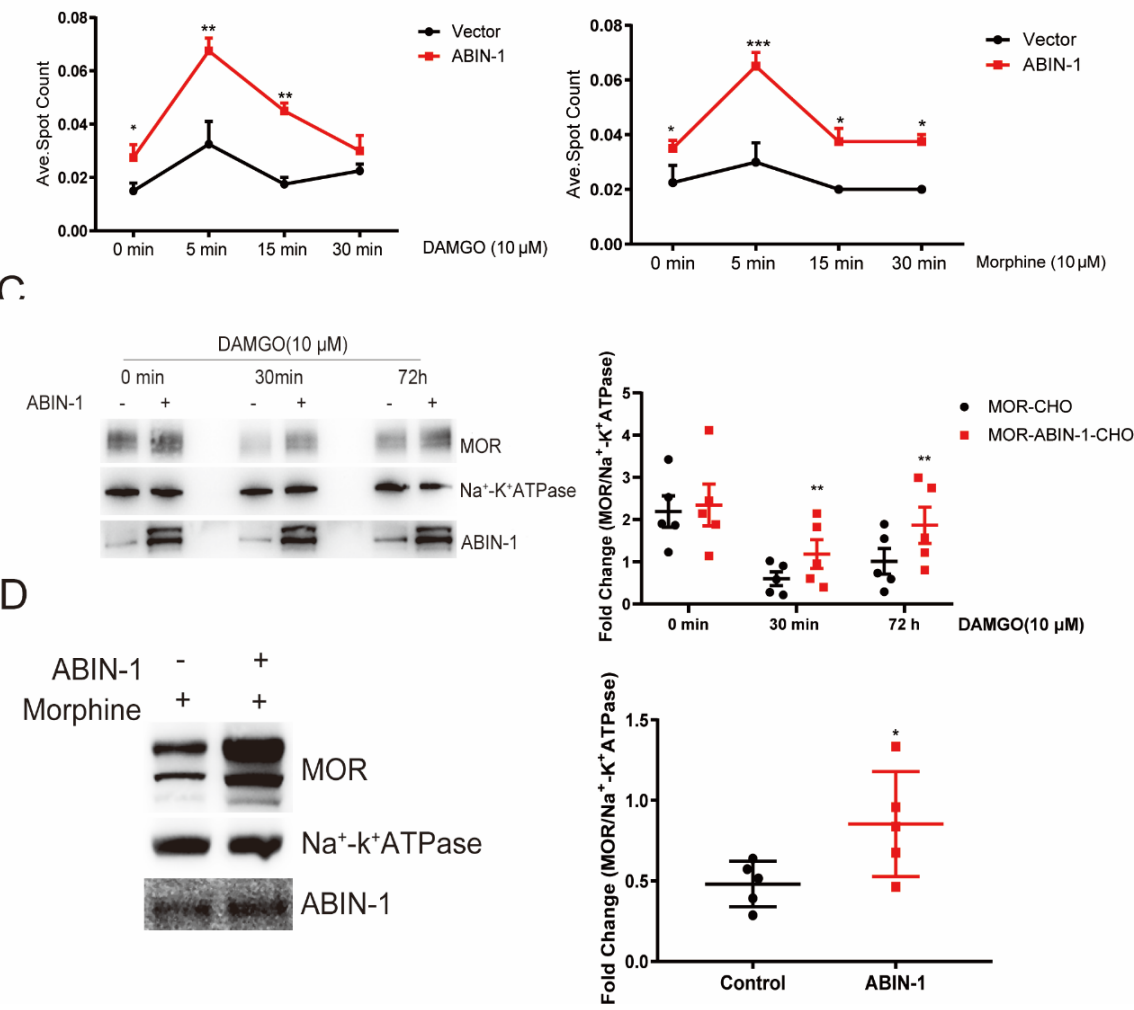

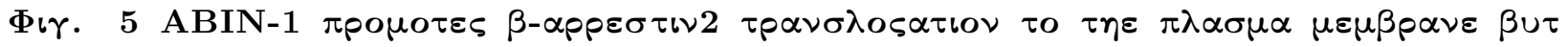

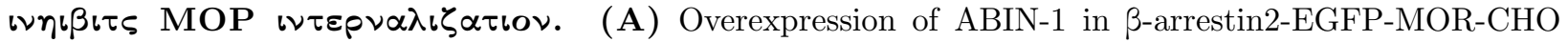
cells was analysed by immunoblotting.(B) $\beta$-arrestin 2 translocation to the plasma membrane was detected by HCS after stimulation with morphine or DAMGO for $0,5,15$, and $30 \mathrm{~min}$. (C) The MOR on plasma membrane in MOR-CHO and MOR-ABIN-1-CHO cells was analysed by immunoblotting under DAMGO $(10 \mu \mathrm{M})$ treatment for $30 \mathrm{~min}$ or $72 \mathrm{~h}$. (D) The MOR on plasma membrane in morphine tolerant mice brain (Hipp) was analysed by immunoblotting after ABIN- overexpression. Two-way ANOVA followed by Bonferroni post hoc test, ABIN-1 vs control, ${ }^{*} P<0.05,{ }^{*} P<0.01,{ }^{* * *} P<0.001 ; \mathrm{n}=5$. The error bars indicate the means \pm S.E.Ms. 
A
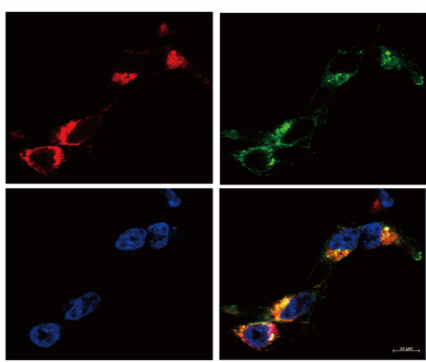

C
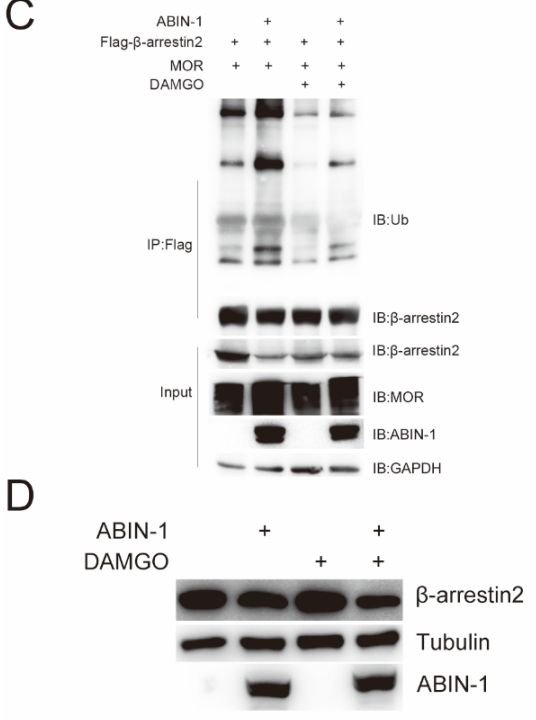

E

Control

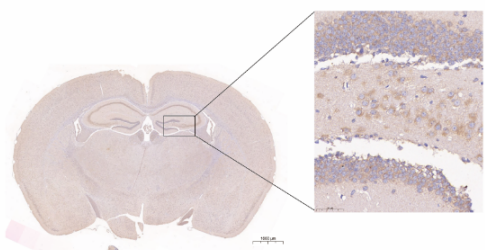

$\mathrm{ABIN}-1$

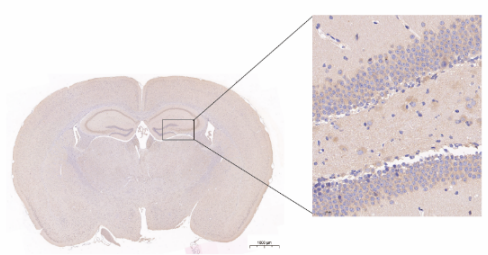

B

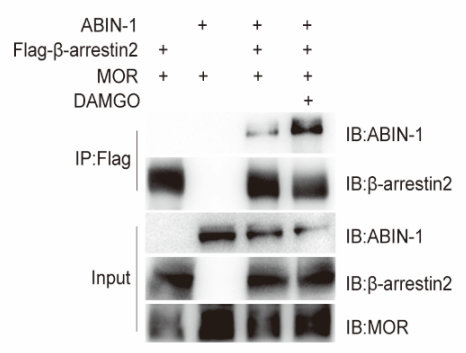

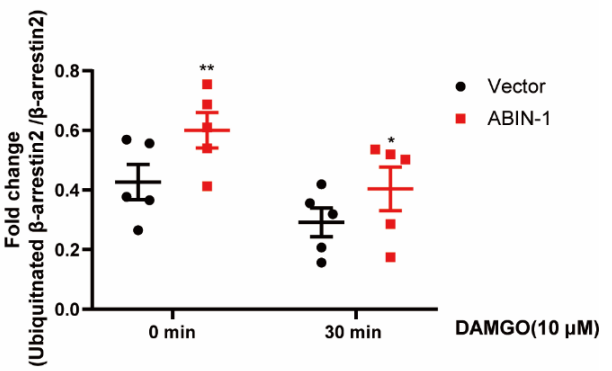
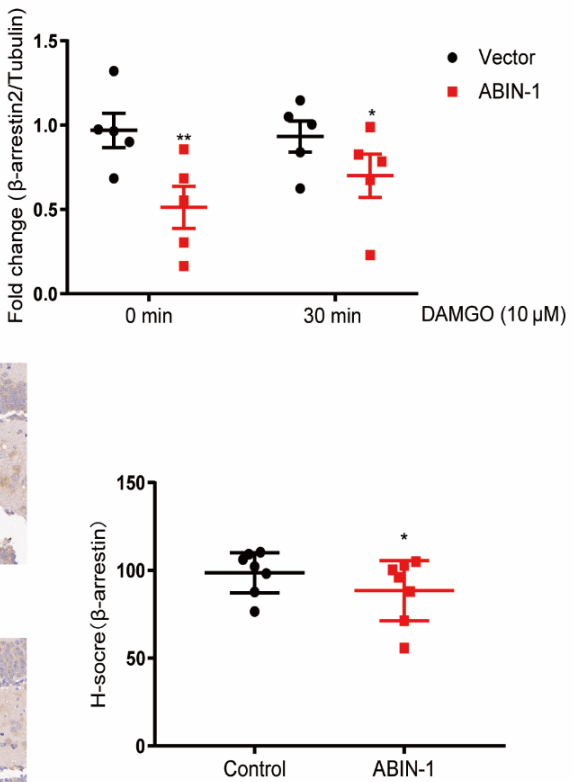

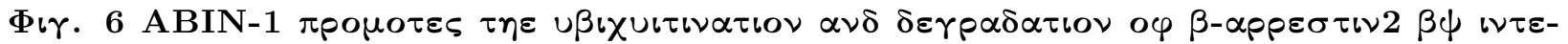

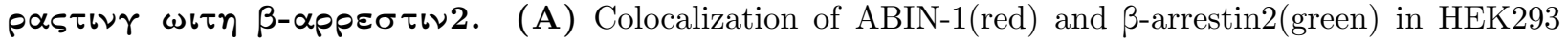
cells. Scale bar, $10 \mu \mathrm{m}$, Pearson's R value: 0.74 (B) The interaction between ABIN-1 and $\beta$-arrestin2 was analysed by co-immunoprecipitation with or without DAMGO $(10 \mu \mathrm{M})$ treatment. (C) The ubiquitination of $\beta$-arrestin2 in HEK293 cell was analysed by co-immunoprecipitation after ABIN-1 overexpression under DAMGO $(10 \mu \mathrm{M})$ treatment. (D) The expression of $\beta$-arrestin2 in Neuro $2 \mathrm{~A}$ cells was analysed by immunoblotting after ABIN-1 overexpression under DAMGO $(10 \mu \mathrm{M})$ treatment. (E) The expression of $\beta$-arrestin2 in morphine tolerant mice (Hipp) was analysed by immunohistochemistry after ABIN-1 overex- 
pression. Student's t test, ABIN-1 vs control, ${ }^{*} P<0.05,{ }^{* *} P<0.01 ; \mathrm{n}=5$. The error bars indicate the means \pm S.E.Ms.

\section{Figure7}

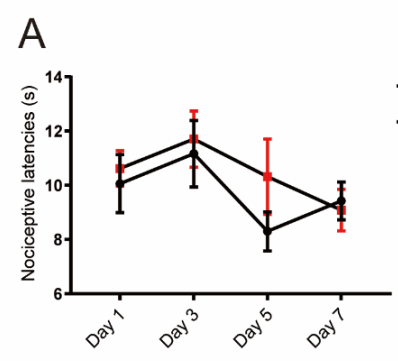

C

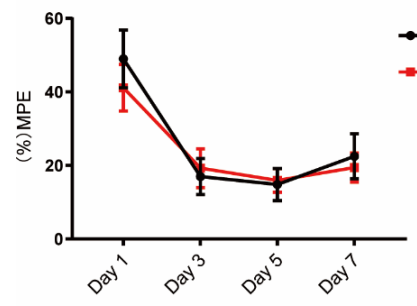

$E$

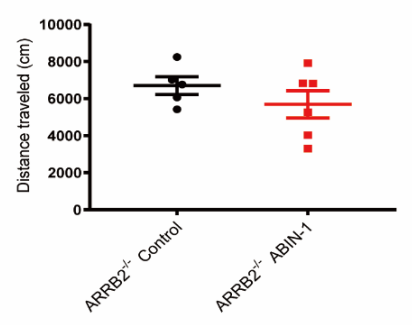

B

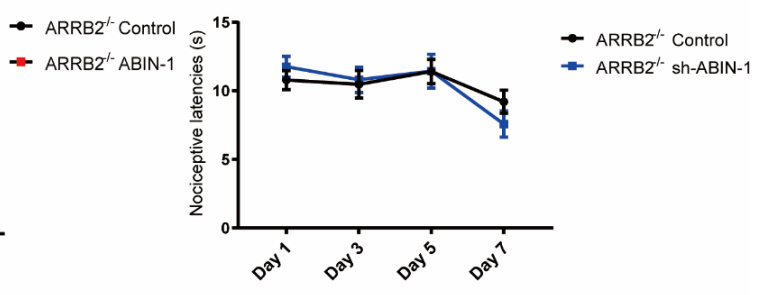

D

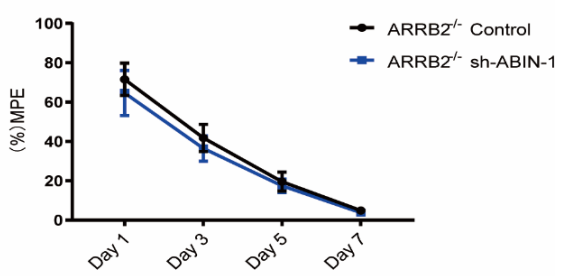

$\mathrm{F}$

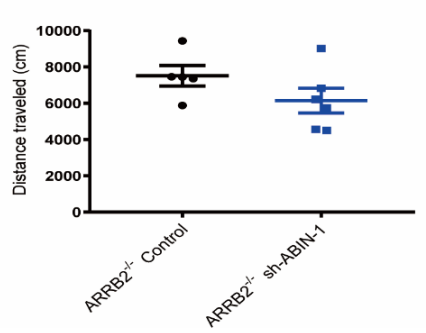

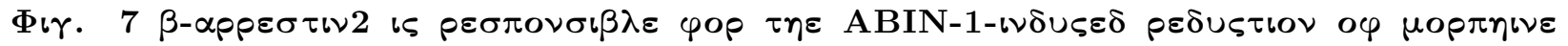

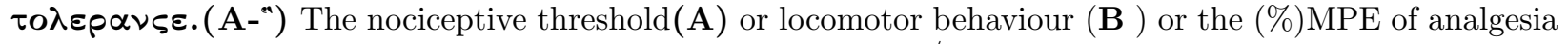
induced by chronic morphine treatment in the brains of $A R R B 2^{-/-}$mice after overexpression the ABIN-1(C) (D-F) The nociceptive threshold (D) or locomotor behaviour (E ) or the (\%)MPE of analgesia induced by chronic morphine treatment in the brains of $A R R B 2^{-/-}$mice after knockdown the ABIN-1 (F). Two-way ANOVA followed by Bonferroni post hoc test ,ABIN-1 vs control, $\mathrm{n}=7-8$. The error bars indicate the means \pm S.E.Ms. 


\section{A}
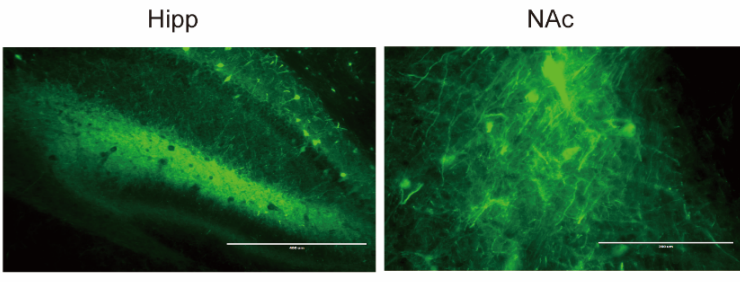

B

C
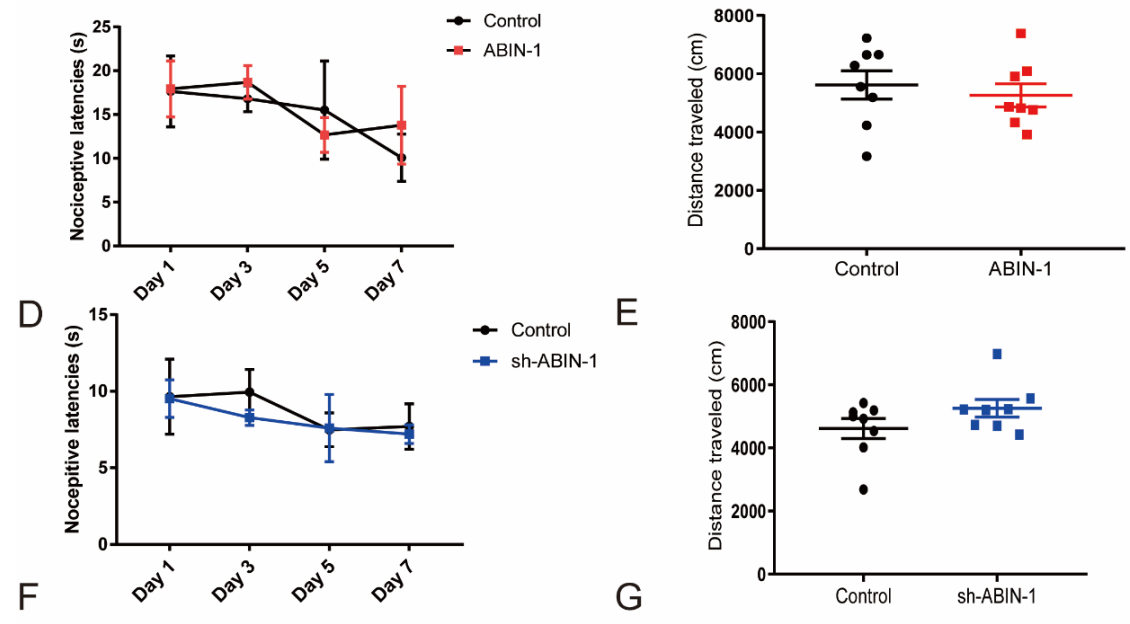

$E$
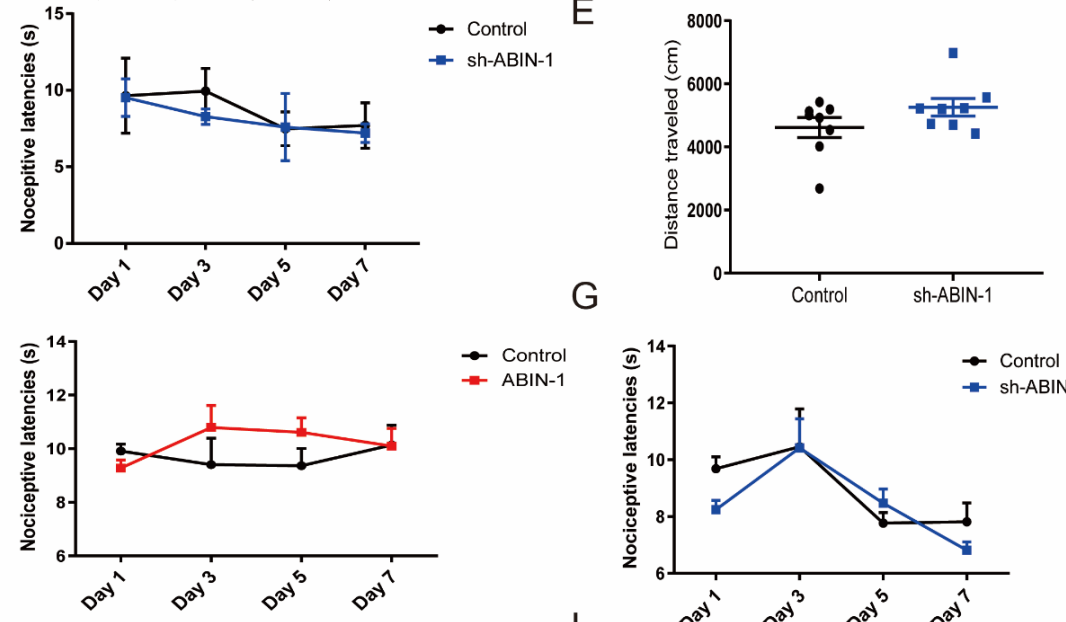

$\rightarrow$ Control
- ABIN-1

$\mathrm{H}$
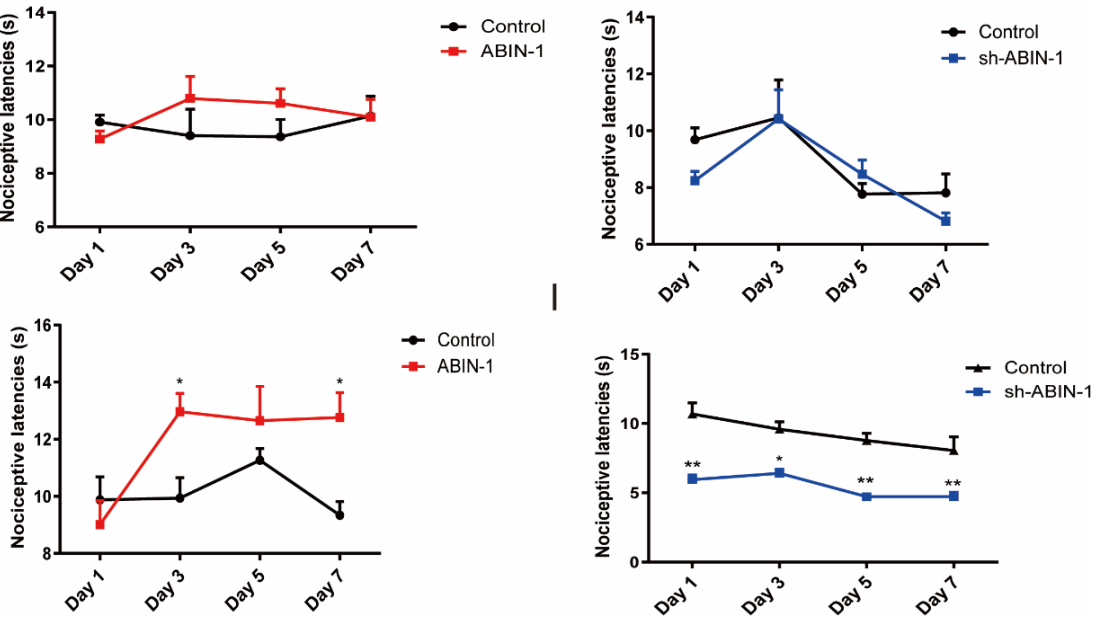

Supplement 1. The nociceptive latencies and locomotor behaviour of mice were measured by a hot plate test before morphine treatment. (A) EGFP-labelled neurons in the hippocampus (Hipp) and nucleus accumbens (NAc) and anterior cingulate cortex (ACC) after injection (i.c.v.) of AAV-ABIN1-virus, bar: $200 \mu \mathrm{m}$.(B-C) The nociceptive latency (B) and locomotor behaviour (C) in mice brain after ABIN-1 overexpression. (D-E) The nociceptive latency(D) and locomotor behaviour (E) in the mice brain after ABIN-1 knockdown. (F-G) The nociceptive latency in the mice hippocampus brain after ABIN-1 overexpression (F) or knockdown (G) . (H-I) The nociceptive latency in the mice nucleus accumbens brain after ABIN-1 overexpression (H) or knockdown (I) . Two-way ANOVA followed by Bonferroni post hoc test, ABIN-1 vs control, ${ }^{*} P<0.05,{ }^{* *} P<0.01 ; \mathrm{n}=7-9$. The error bars indicate the means \pm S.E.Ms. 


\section{Supplement3}

A

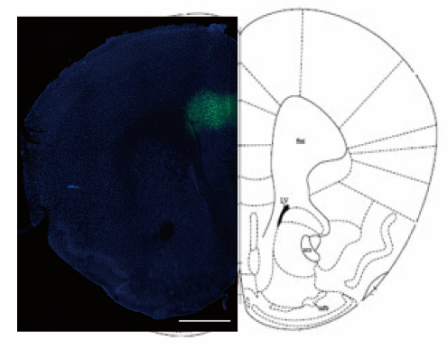

B

$\mathrm{C}$
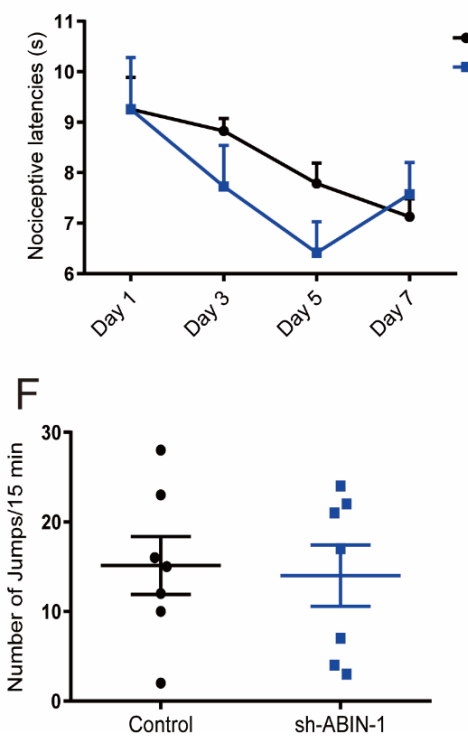

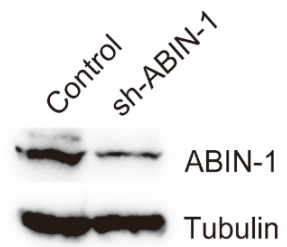

$E$

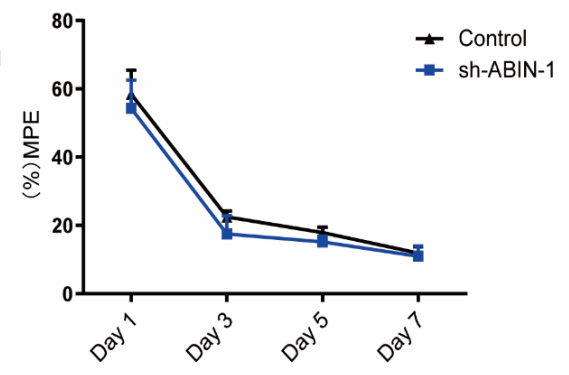

Supplement 2. ABIN-1 in the anterior cingulate cortex (ACC) has no effect on morphine tolerance. (A) EGFP-labelled neurons in the ACC. Blue, DAPI. Bar: 1,000 $\mu \mathrm{m}$. (B) The expression of ABIN-1 in the ACC was interfered by injection AAV-shRNA-ABIN-1.(D-F) The nociceptive threshold (D) or the (\%)MPE of analgesia effects(E) or the jumping counts of naloxone-precipitated withdrawal(F) induced by chronic morphine treatment in the mice anterior cingulate cortex brain after ABIN-1 knockdown. Two-way ANOVA followed by Bonferroni post hoc, ABIN-1 vs control, $\mathrm{n}=6-8$. The error bars indicate the means \pm S.E.Ms. 
Figure1

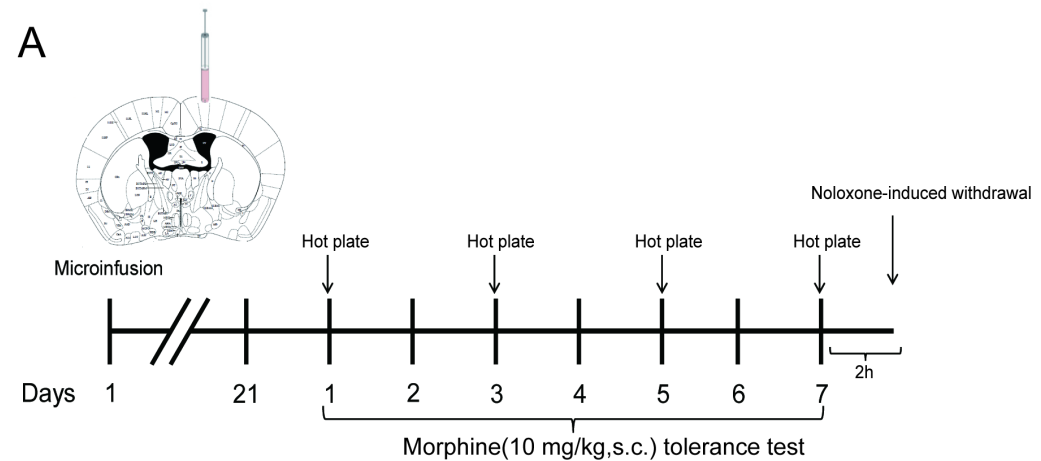

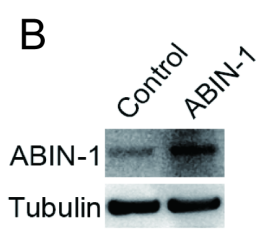

D

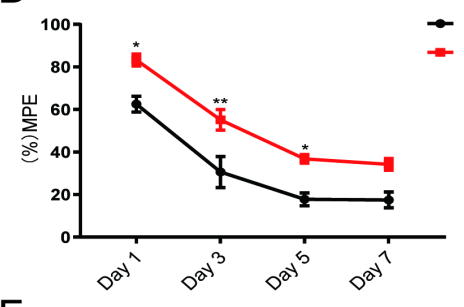

F

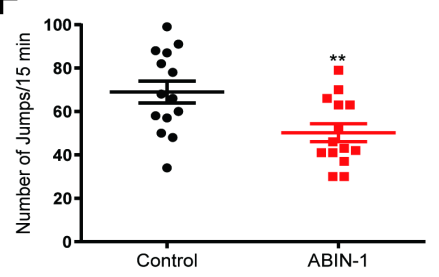

C
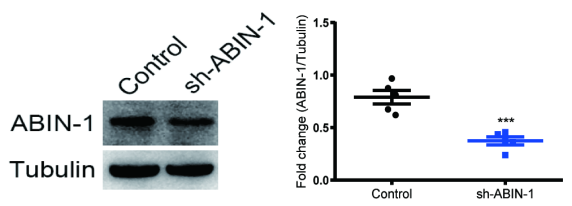

E

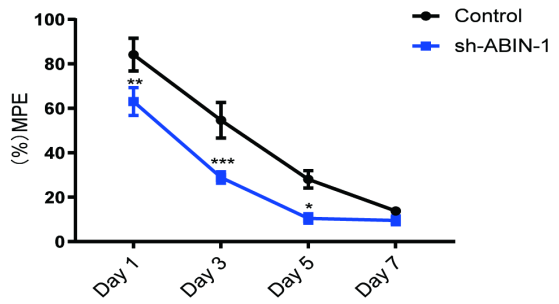

G

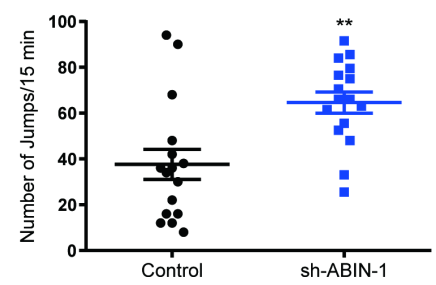


Figure2

A

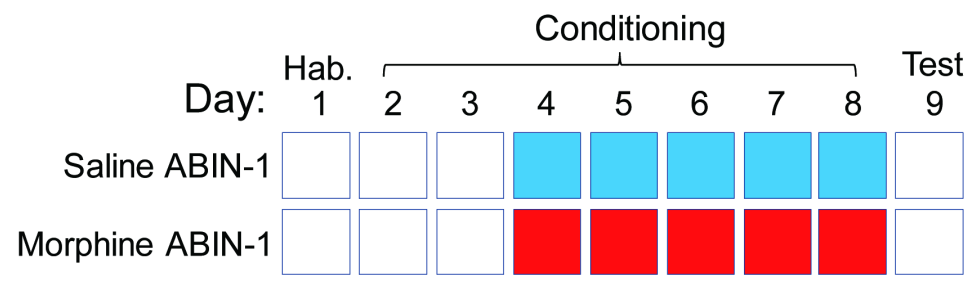

Free access to both chambers

Saline in conditioned chamber

Morphine $(10 \mathrm{mg} / \mathrm{kg})$ in conditioned chamber

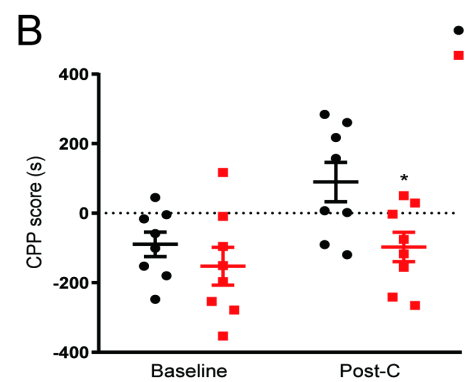

C

ABIN-1

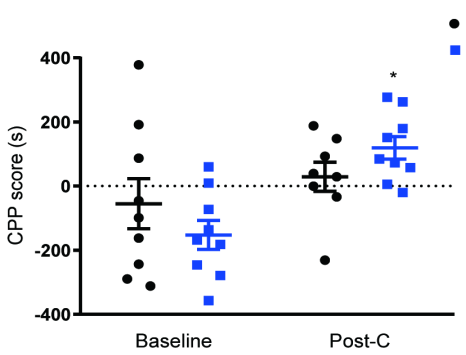


A
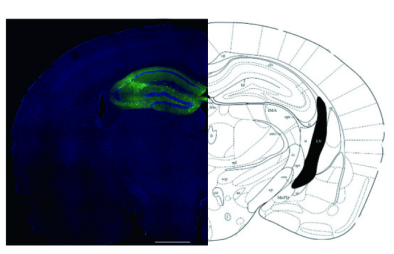

C
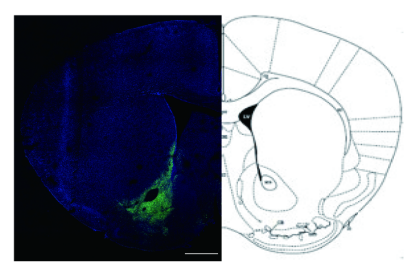

E
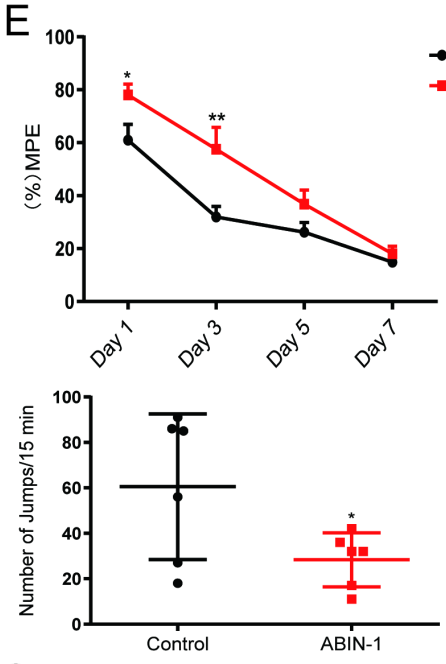

G
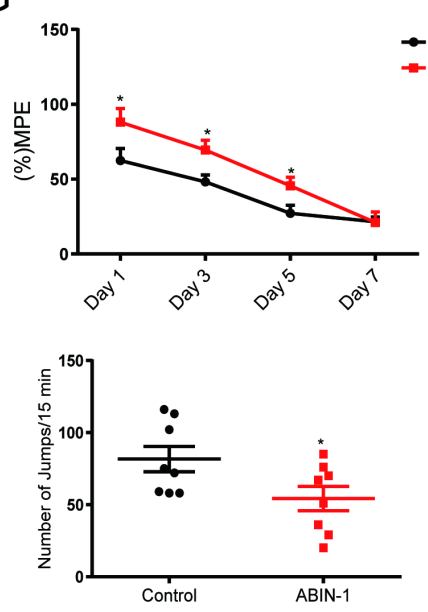

B

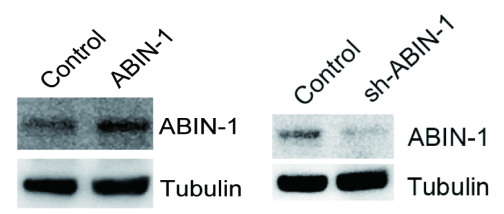

D

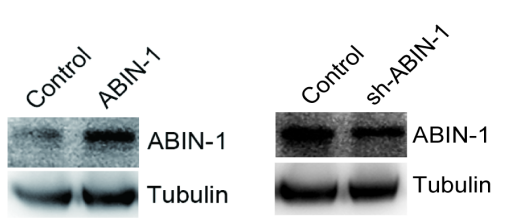

$\mathrm{F}$

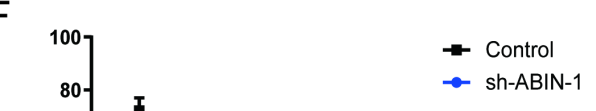

$\mathrm{H}$
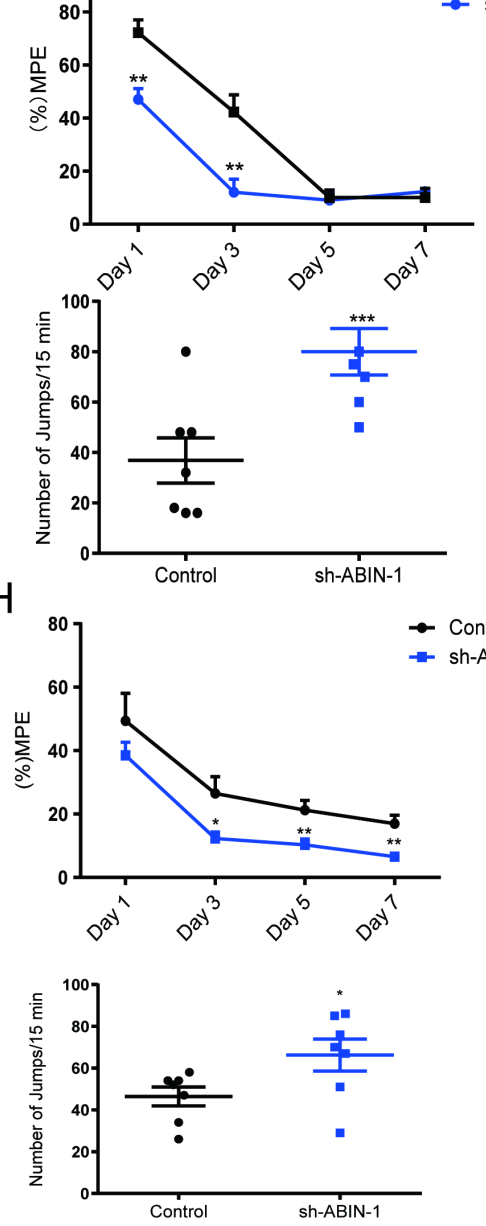
A

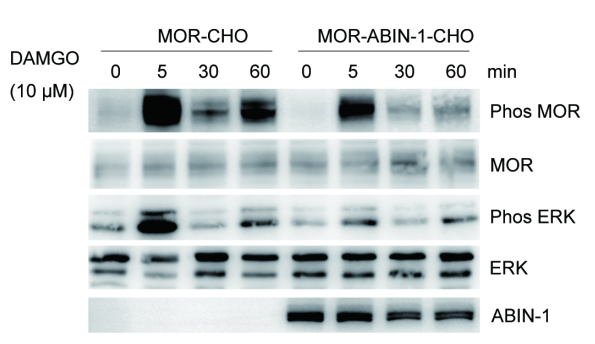

B

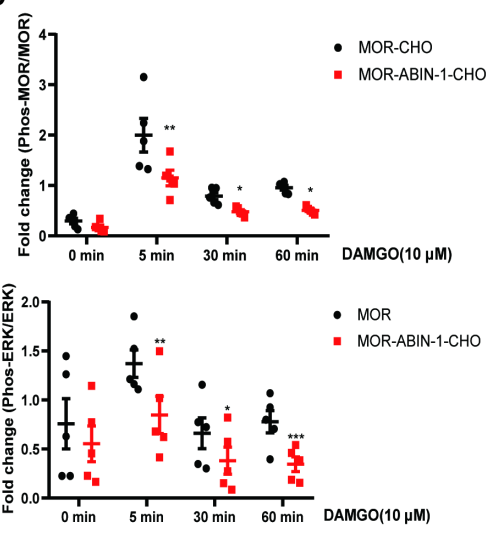

C

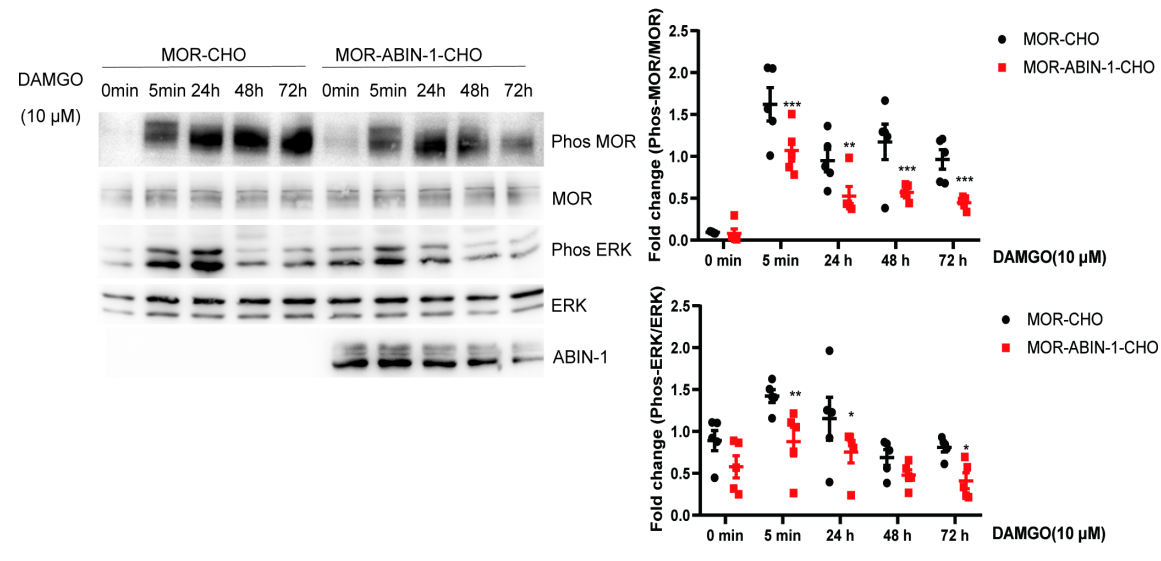


A

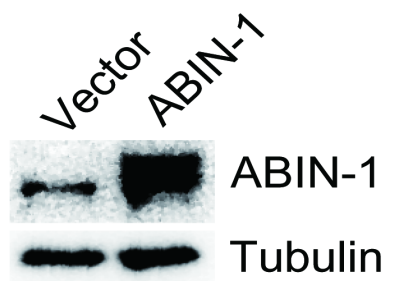

B
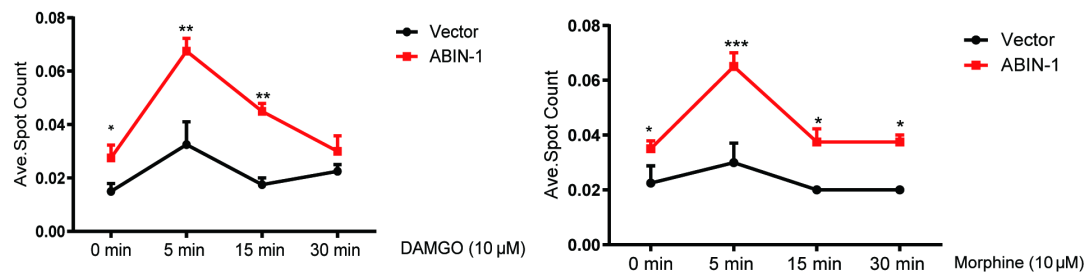

r.
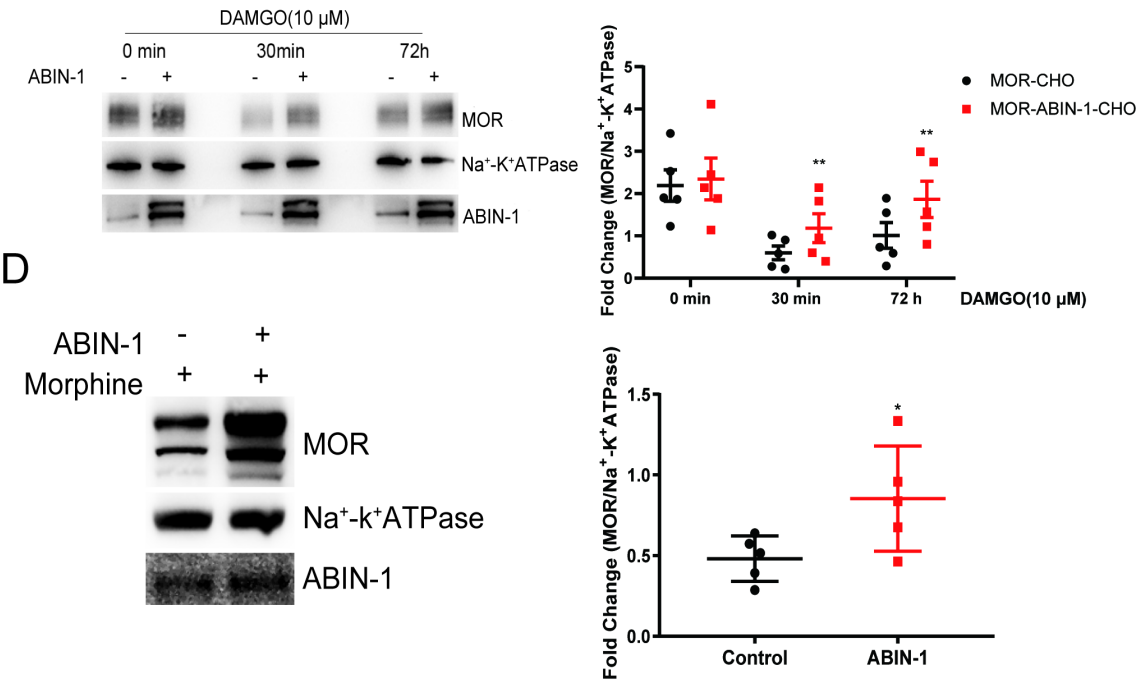
A
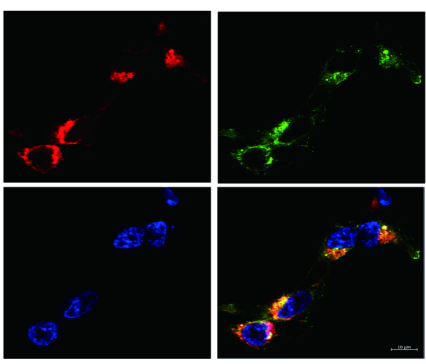

C

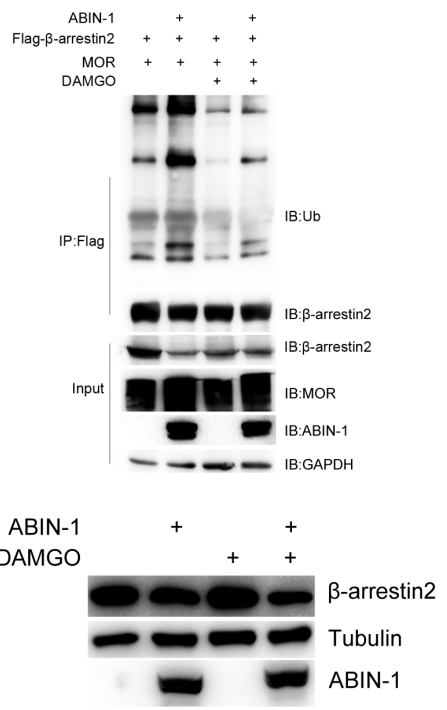

E

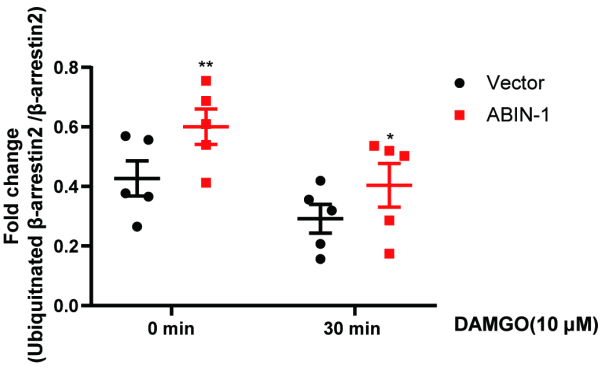

D

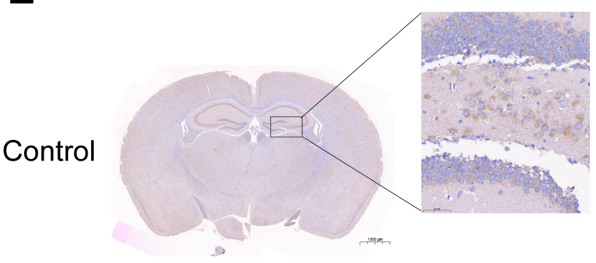

$\mathrm{ABIN-1}(1)$
B

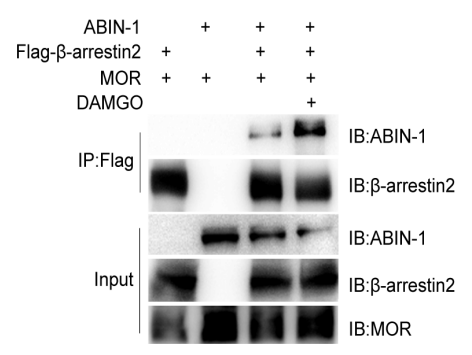

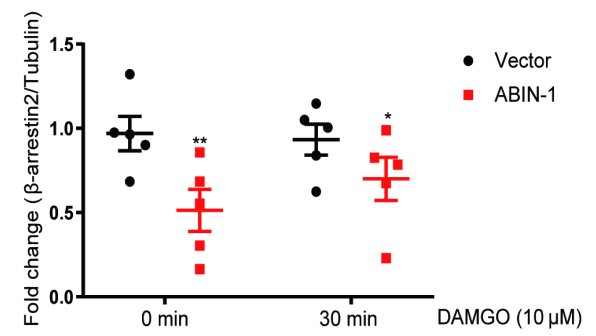

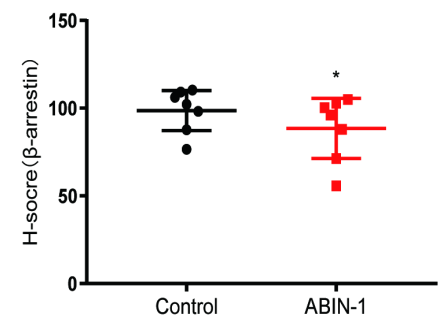


Figure7

A B

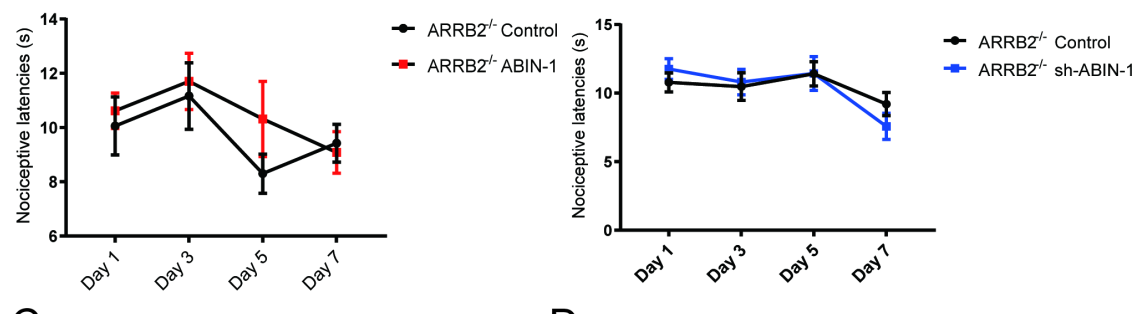

C

D
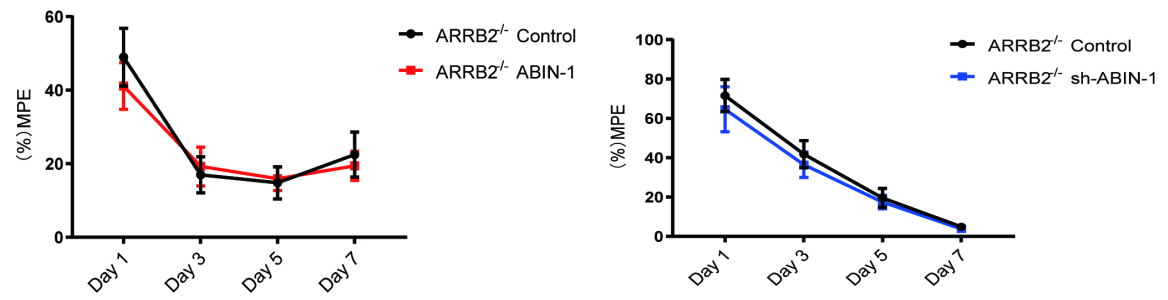

E

$\mathrm{F}$
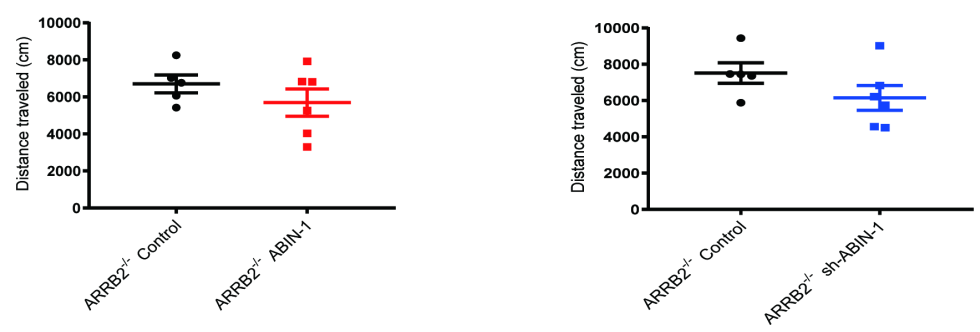
A
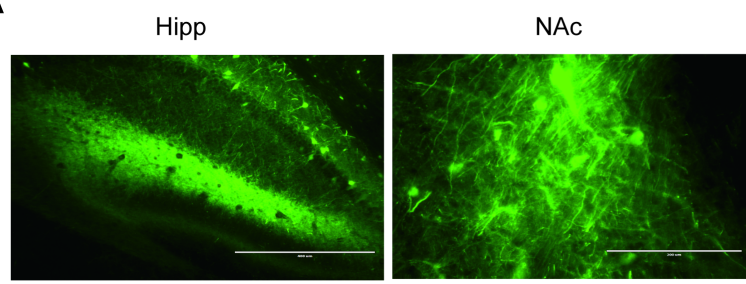

B

C

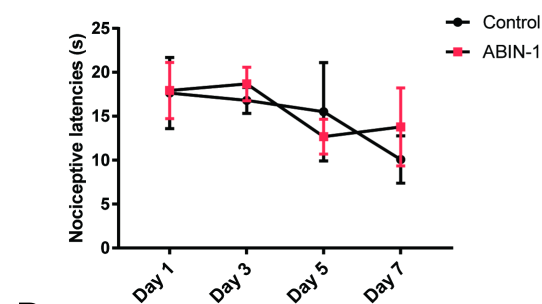

D
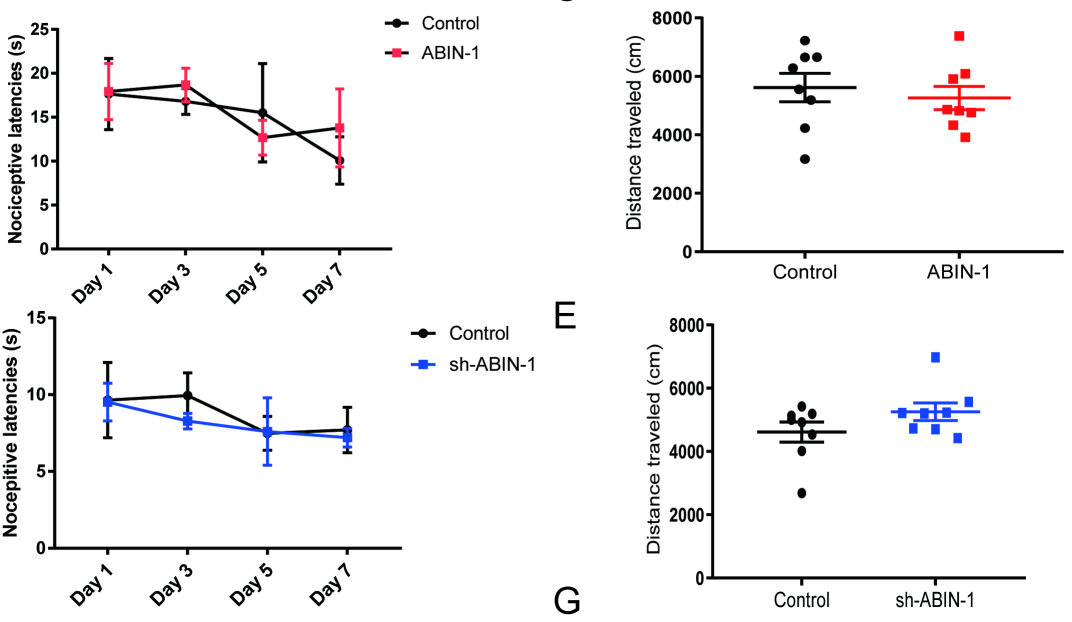

E
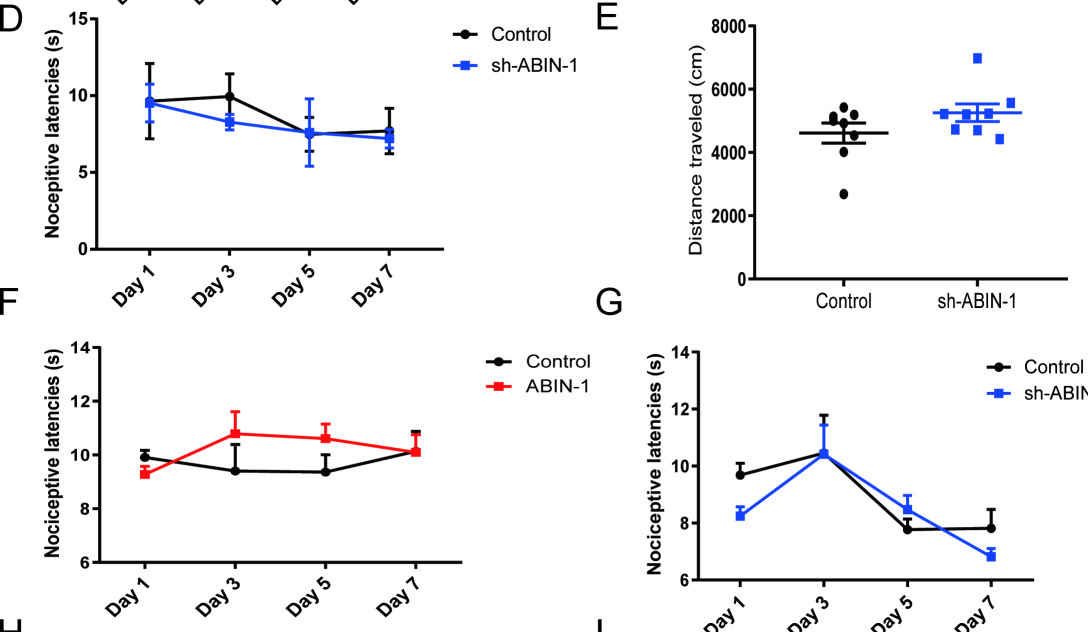

- Control
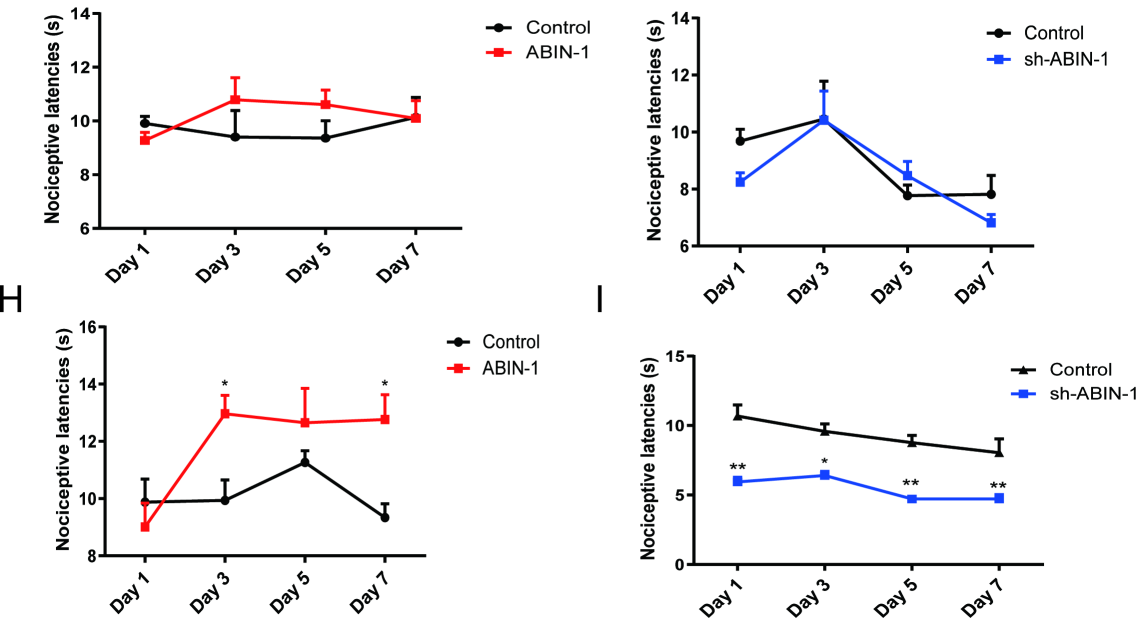
Supplement3

A

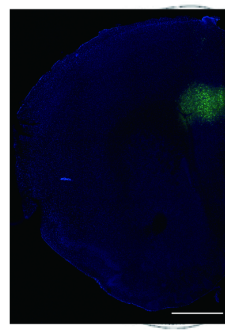

C

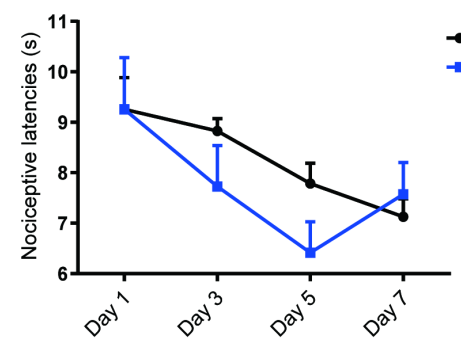

$\mathrm{F}$

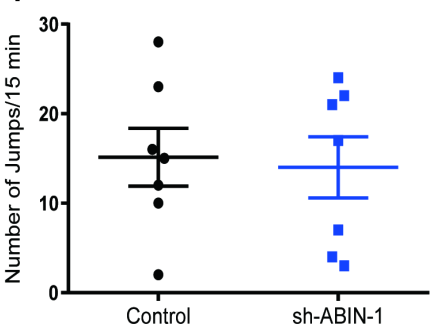

B

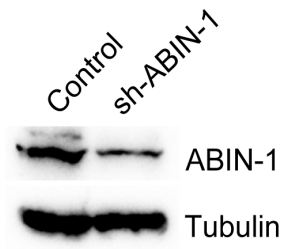

E

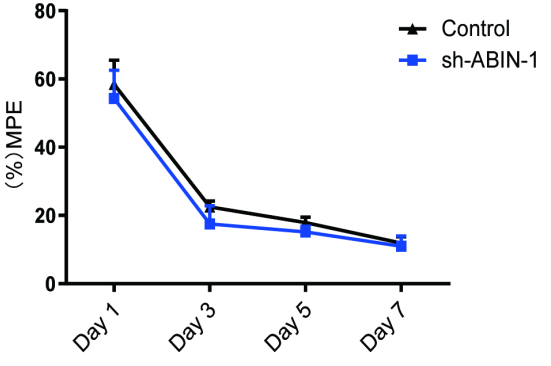

